\title{
AN ADVANCED LEAKAGE SCHEME FOR NEUTRINO TREATMENT IN ASTROPHYSICAL SIMULATIONS
}

\author{
A. Perego ${ }^{1}$, R. M. Cabezón ${ }^{2}$, and R. KÄPPeli ${ }^{3}$ \\ ${ }^{1}$ Institut für Kernphysik, Technische Universität Darmstadt, Schlossgartenstraße 2, D-64289 Darmstadt, Germany; albino.perego@physik.tu-darmstadt.de \\ ${ }^{2}$ Physics Department, University of Basel, Klingelbergstrasse 82, CH-4056 Basel, Switzerland \\ ${ }^{3}$ Seminar for Applied Mathematics, ETH Zürich, Rämistrasse 101, 8092 Zürich, Switzerland \\ Received 2015 November 20; accepted 2016 February 19; published 2016 April 12
}

\begin{abstract}
We present an Advanced Spectral Leakage (ASL) scheme to model neutrinos in the context of core-collapse supernovae (CCSNe) and compact binary mergers. Based on previous gray leakage schemes, the ASL scheme computes the neutrino cooling rates by interpolating local production and diffusion rates (relevant in optically thin and thick regimes, respectively) separately for discretized values of the neutrino energy. Neutrino trapped components are also modeled, based on equilibrium and timescale arguments. The better accuracy achieved by the spectral treatment allows a more reliable computation of neutrino heating rates in optically thin conditions. The scheme has been calibrated and tested against Boltzmann transport in the context of Newtonian spherically symmetric models of CCSNe. ASL shows a very good qualitative and a partial quantitative agreement for key quantities from collapse to a few hundreds of milliseconds after core bounce. We have proved the adaptability and flexibility of our ASL scheme, coupling it to an axisymmetric Eulerian and to a three-dimensional smoothed particle hydrodynamics code to simulate core collapse. Therefore, the neutrino treatment presented here is ideal for large parameter-space explorations, parametric studies, high-resolution tests, code developments, and long-term modeling of asymmetric configurations, where more detailed neutrino treatments are not available or are currently computationally too expensive.
\end{abstract}

Key words: hydrodynamics - neutrinos - radiative transfer - stars: neutron - supernovae: general

\section{INTRODUCTION}

Neutrinos are elusive, weakly interacting particles. Owing to their small cross sections with ordinary matter, which make their detection on Earth so challenging, they represent a very efficient way for astrophysical dense and hot plasma to radiate energy away. In particular, they are expected to be copiously emitted in stellar explosive scenarios, including core-collapse supernovae (CCSNe; e.g., Janka 2012; Kotake et al. 2012a; Burrows 2013; Foglizzo et al. 2015, for recent reviews) and compact binary mergers (e.g., Shibata \& Taniguchi 2011; Faber \& Rasio 2012; Rosswog 2015b, for recent reviews). The modeling of such systems is extremely stimulating, owing to the large variety of involved scales and to the complex and rich physics required. The treatment of neutrinos, in particular their transport from optically thick to optically thin regions, is among the most crucial and difficult parts to model. This is even more evident for intrinsically multidimensional problems, where the solution of the Boltzmann transport equation would result in a genuine seven-dimensional problem (e.g., Lindquist 1966).

The large variety of questions and possible initial conditions, together with the parallel increase of computational power, have motivated the development of several neutrino treatments, which differ in complexity and accuracy. The solution of the complete Boltzmann equation for neutrino radiation has been performed for spherically symmetric simulations of CCSNe (Mezzacappa \& Bruenn 1993a, 1993b, 1993c; Liebendörfer et al. 2004; Sumiyoshi et al. 2005). Solutions in 2D (e.g., Livne et al. 2004; Ott et al. 2008; Brandt et al. 2011) and, recently, in three dimensions (Sumiyoshi \& Yamada 2012; Sumiyoshi et al. 2015), neglecting velocity-dependent terms, in the context of collapsing stellar cores, have also been presented. Lately, Nagakura et al. (2014) proposed a novel numerical method for solving special relativistic Boltzmann equations for neutrinos coupled to hydrodynamics equations. The velocity-dependent term is fully included in it. This method has been tested in spherically symmetric CCSN simulations, and it can be potentially extended to multidimensional models. Another sophisticated approach to the neutrino transport problem is represented by the so-called Moment schemes. In these schemes, the explicit angular dependence in the neutrino momentum space is removed by integrating the distribution function and introducing momenta (for example, the energy density is the zeroth momentum, while the linear momentum density is the first momentum). The Boltzmann transport equation is replaced by time evolution equations for the different momenta. Among them, we recall the (multigroup) flux-limited diffusion schemes, (MG) FLD, where only the zeroth moment is considered (Arnett 1977; Bowers \& Wilson 1982; Bruenn 1985; Swesty \& Myra 2009; Zhang et al. 2013; for related applications see, e.g., Fryer 1999; Dessart et al. 2006, 2009; Burrows et al. 2007; Yakunin et al. 2010; Bruenn et al. 2013; Dolence et al. 2015), and the M1 schemes, where both the zeroth and first moments are taken into account (see, e.g., Pons et al. 2000; Kuroda et al. 2012; O'Connor \& Ott 2013; Obergaulinger et al. 2014; Foucart et al. 2015; Just et al. 2015; O'Connor 2015). The closure relation in M1 schemes is usually provided by an analytic expression. It is possible to design moment schemes where the closure relation is not analytic, but it is given by a variable Eddington tensor (e.g., Burrows et al. 2000; Rampp \& Janka 2002; Thompson et al. 2003; Buras et al. 2006a, 2006b; Müller et al. 2010; Tamborra et al. 2013). The latter solution is close to the solution of the full Boltzmann equation. Another noteworthy approximate transport scheme is the isotropic diffusion source approximation (IDSA, Liebendörfer et al. 2009; see Suwa et al. 2011; Nakamura et al. 2014; Takiwaki et al. 2014; Pan et al. 2015; Suwa et al. 2015 for some recent applications), where the 
distribution function is separated into a trapped and a freestreaming component. Also Monte Carlo methods have been developed in the context of neutrino transport (see, e.g., Janka \& Hillebrandt 1989a, 1989b; Janka 1992; Abdikamalov et al. 2012; Richers et al. 2015). More approximate treatments include gray transport schemes (Scheck et al. 2006), gray neutrino leakage schemes (Ruffert et al. 1996; Rosswog \& Liebendörfer 2003), and lightbulb schemes (Murphy \& Burrows 2008; Fernández 2012; Hanke et al. 2012; Couch \& Ott 2013; Fernández \& Metzger 2013; Handy et al. 2014).

In this paper, we present an improved and more sophisticated version of the classical leakage scheme. Leakage schemes have a long history in computational astrophysics: owing to their reduced computational cost and to their flexibility, they were, after FLD schemes, among the first developed neutrino treatments for spherically symmetric core-collapse models (van Riper \& Lattimer 1981; Bludman et al. 1982; Cooperstein et al. 1986) and for the first three-dimensional (3D) compact binary merger simulations (Ruffert et al. 1996; Ruffert \& Janka 1999; Rosswog \& Liebendörfer 2003). More recently, gray leakage schemes have been widely applied to study, for example, (i) neutrino and gravitational wave emissions from rotating and/or magnetized collapsing stellar cores, in axisymmetry (Kotake et al. 2005, 2012b) or in three dimensions (Scheidegger et al. 2010; Takiwaki \& Kotake 2011); (ii) SN explosions caused by the magnetorotational mechanism (e.g., Suwa et al. 2007; Takiwaki et al. 2009; Winteler et al. 2012); (iii) the influence of the pions and hyperons in the nuclear equation of state (EOS) of stellar cores collapsing into a black hole (Peres et al. 2013); (iv) the impact of asphericity in the progenitor model for core-collapse simulations (Couch \& Ott 2013); and (v) gravitational waves and neutrino emission from Newtonian simulations of compact binary mergers (Rosswog et al. 2013). General relativistic extensions of the gray scheme have also been developed (O’Connor \& Ott 2010; Sekiguchi 2010; Galeazzi et al. 2013). They have been used, for example, to simulate (i) spherically symmetric models of $\mathrm{SNe}$ and black hole formation from massive stellar cores (O'Connor \& Ott 2011); (ii) 3D general relativistic core-collapse models (Ott et al. 2013), including also magnetic fields (Mösta et al. 2014); (iii) gravitational waves and neutrino emission from general relativistic simulations of compact binary mergers (Kiuchi et al. 2012; Deaton et al. 2013; Sekiguchi et al. 2015); and (iv) the collapse of a rotating stellar core to a black hole surrounded by an accretion disk (Sekiguchi 2011).

Despite such a broad application field, detailed comparisons between the results obtained by a leakage scheme and more sophisticated neutrino transports are difficult to find. For example, Dessart et al. (2009) and Foucart et al. (2015, 2016) compared the luminosities obtained by a gray leakage scheme with the ones obtained by MGFLD or moment schemes, during and after a compact binary merger. Moreover, O'Connor \& Ott (2010) and Sekiguchi (2010) provide temporal profiles of the neutrino luminosities and mean energies occurring after core bounce in CCSN simulations, together with a few radial profiles of some relevant quantities (e.g., entropy or electron fraction). These results can be compared with reference results in the literature (e.g., Liebendörfer et al. 2005). They found a qualitative good agreement, even if quantitative differences were present. This confirms the idea that leakage schemes capture the dominant aspects of neutrino cooling, even if the evolution of the neutrino field in the opaque region and the inclusion of consistent absorption terms in the optically thin region remain challenging.

In the Advanced Spectral Leakage (ASL) treatment we conjugate the usual positive aspects associated with leakage schemes (mainly, the reduced computational cost and flexibility), with an improved accuracy, obtained using a spectral approach (i.e., solving different leakage schemes, for different energy bins), modeling a neutrino trapped component in the optically thick region, and including a consistent absorption term obtained from the spectral cooling rates. The development of the ASL treatment has been performed in the framework of spherically symmetric models of CCSNe, where the new treatment has been compared against a detailed Boltzmann neutrino transport. Perego et al. (2015) used it, in combination with IDSA for electron flavor neutrinos, to model heavy flavor neutrinos in spherically symmetric, artificially induced explosions of CCSNe. However, one of the goals of this approximate scheme is the application to multidimensional models, with reduced computational costs. Applications of the ASL scheme in multidimensional astrophysical simulations have been already performed in the past few years. Winteler et al. (2012) simulated a magnetically driven CCSN explosion of a $15 M_{\odot}$ progenitor star, using the 3D, Cartesian MHD code FISH (Käppeli et al. 2011) coupled with a previous version of the ASL scheme to model the neutrino cooling. Perego et al. (2014b) studied the neutrino-driven wind that emerges from the remnant of a binary neutron star merger, in the presence of a long-lived massive neutron star. They used the FISH code coupled with the ASL scheme. The neutrino heating rates were based on neutrino densities in optically thin regions, computed by a ray-tracing algorithm.

In Section 2, we provide a detailed presentation of the ASL scheme and of its terms. In Section 3, we test the new scheme in the context of spherically symmetric models of CCSNe, comparing the results obtained by the new treatment with the solution of the Boltzmann transport equation. We also briefly explore the impact of the variation of the few free parameters present in the scheme. In Section 4, we show the flexibility and the versatility of the ASL scheme by implementing it in two different multidimensional codes, a grid code and a smoothed particle hydrodynamics (SPH) code, modeling CCSNe. Finally, in Section 5, we summarize and discuss our results.

\section{THE ASL TREATMENT}

\subsection{Neutrino Description and Interactions}

The ASL scheme is an approximate neutrino treatment designed for neutrinos and antineutrinos of all flavors. While electron neutrinos $\left(\nu_{e}\right)$ and antineutrinos $\left(\bar{\nu}_{e}\right)$ are considered separately, $\mu$ and $\tau$ neutrinos, as well as their antiparticles, are treated as a single neutrino species $\left(\nu_{\mu, \tau}\right)$. For each of the three independent species, we perform a spectral treatment, i.e., we distinguish between neutrinos with different energies.

The interaction between matter and neutrinos is provided by weak interaction processes. For each production, absorption, or scattering reaction, we compute the corresponding spectral emissivity $j_{\nu}(E, \boldsymbol{x})$, absorptivity $\chi_{\nu, \mathrm{ab}}(E, \boldsymbol{x})$, or scattering rate $\chi_{\nu, \mathrm{sc}}(E, \boldsymbol{x})$, respectively, as they are defined in the Boltzmann transport equation, for a neutrino energy $E$ and a position $\boldsymbol{x}$ (in this section we consider a fixed time $t$ ). The total emissivity and absorptivity are computed as the sum over all considered 
neutrino processes. While the emissivity provides the local rates of neutrino production, the absorptivity and the scattering rates are the sources of the local neutrino opacity. The local opacity can be expressed in terms of the total mean free path $\lambda_{\nu, \text { tot }}$,

$$
\lambda_{\nu, \text { tot }}(E, \boldsymbol{x})=c\left(\sum_{r} \chi_{\nu, \mathrm{ab}, r}(E, \boldsymbol{x})+\sum_{s} \chi_{\nu, \mathrm{sc}, s}(E, \boldsymbol{x})\right)^{-1},
$$

where $c$ is the speed of light, and the indexes $r$ and $s$ run over all the considered absorption and scattering reactions, respectively. Besides the total mean free path, in which all reactions are treated equally, we define also an energy mean free path, $\lambda_{\nu}$, en. The latter represents the mean free path over which neutrinos can effectively exchange energy with the fluid. To compute it, we perform the geometrical mean between the total mean free path and the mean free path only due to highly inelastic processes, i.e., all the absorption processes and the scattering processes where the energy of the incoming and outgoing neutrinos is expected to differ significantly ${ }^{4}$ (see, e.g., Shapiro \& Teukolsky 1986 or Raffelt 2001 for analogous expressions):

$$
\lambda_{\nu, \text { en }}(E, \boldsymbol{x})=\left(c^{-1} \sum_{s^{\prime}} \chi_{\nu, \text { inel }, s^{\prime}}(E, \boldsymbol{x})\right)^{-1 / 2}\left(\lambda_{\nu, \text { tot }}(E, \boldsymbol{x})\right)^{1 / 2},
$$

where we have restricted the sum only over inelastic processes, abbreviated by inel and labeled by $s^{\prime}$.

The neutrino optical depth $\tau_{\nu}$ is defined as the path integral of the inverse neutrino mean free path, $\lambda_{\nu}^{-1}$, calculated on a typical radiation path, $\gamma$, connecting any point $\boldsymbol{x}$ of the system with its edge:

$$
\tau_{\nu, \gamma}(E, \boldsymbol{x})=\int_{\gamma: \boldsymbol{x} \rightarrow+\infty} \frac{1}{\lambda_{\nu}\left(E, \boldsymbol{x}^{\prime}(s)\right)} d s .
$$

From a physical point of view, it is a measure of the accumulated opacity of matter to radiation along an escape path: it counts the number of interactions that, on average, a radiation particle, emitted at a certain point, experiences before leaving the system. The two mean free paths introduced above can be used to compute two different optical depths, a total optical depth, $\tau_{\nu \text {,tot }}(E, \boldsymbol{x})$, and an energy optical depth, $\tau_{\nu \text {,en }}(E, \boldsymbol{x})$. We note that $\tau_{\nu \text {,en }} \leqslant \tau_{\nu \text {,tot }}$, by definition. In the case of spherically symmetric models, the optical depth retains the spherical symmetry and $\tau_{\nu}$ can be simply calculated along radial paths:

$$
\tau_{\nu}(E, R)=\int_{R}^{+\infty} \frac{1}{\lambda_{\nu}(E, r)} d r .
$$

Otherwise, for more general geometries, multidimensional algorithms are required to compute the optical depth along paths that minimize the number of neutrino-matter interactions (e.g., Perego et al. 2014a).

\footnotetext{
4 Elastic scattering processes enter the definition of $\lambda_{\nu, \text { en }}$ via $\lambda_{\nu, \text { tot }}$. Even if they do not allow direct energy exchange between neutrinos and matter, they still provide opacity and increase the probability of inelastic processes to locally happen.
}

According to the values of $\tau_{\nu, \text { tot }}$ and $\tau_{\nu, \text { en }}$ for the most relevant neutrino energies, several different regimes can be distinguished:

1. $\tau_{\nu, \text { tot }} \gg 1$ and $\tau_{\nu, \text { en }} \gtrsim 1$, the equilibriumdiffusive regime. The radiation field is in thermal and weak equilibrium with the surrounding matter, and neutrinos can be considered as a trapped Fermi gas inside the fluid, behaving like a fluid component itself. Under these assumptions, the distribution functions describing the neutrino gas can be expressed as $f_{\nu}(\boldsymbol{p}, \boldsymbol{x})=f_{\nu}^{\mathrm{tr}}(\boldsymbol{p}, \boldsymbol{x})+\delta f_{\nu}(\boldsymbol{p}, \boldsymbol{x}), \quad$ where $f_{\nu}^{\mathrm{tr}}$ is the trapped component and $\delta f_{\nu}$ is a small deviation from equilibrium, which we neglect (Cooperstein et al. 1986, 1987; Cooperstein 1988). We assume further that the trapped component has no explicit angular dependence in the momentum space, $f_{\nu}^{\mathrm{tr}}(E, \boldsymbol{x})$ (for a more detailed discussion of the decomposition of the neutrino distribution function in an [isotropic] trapped component and a free-streaming one see Liebendörfer et al. 2009). The integration over the neutrino phase space of $f_{\nu}^{\mathrm{tr}}$ and $f_{\nu}^{\mathrm{tr}} E$ gives information about the particle and the energy contents of the neutrino gas:

$$
\begin{aligned}
& Y_{\nu}(\boldsymbol{x})=\frac{4 \pi}{(h c)^{3}} \frac{m_{\mathrm{b}}}{\rho(\boldsymbol{x})} \int f_{\nu}^{\mathrm{tr}}(E, \boldsymbol{x}) E^{2} d E, \\
& Z_{\nu}(\boldsymbol{x})=\frac{4 \pi}{(h c)^{3}} \frac{m_{\mathrm{b}}}{\rho(\boldsymbol{x})} \int f_{\nu}^{\mathrm{tr}}(E, \boldsymbol{x}) E^{3} d E,
\end{aligned}
$$

where $m_{\mathrm{b}}$ is the baryon mass and $h$ the Planck constant.

2. $\tau_{\nu \text {,tot }} \gg 1$, but $\tau_{\nu \text {,en }} \lesssim 1$, the diffusive regime. Neutrinos still diffuse, but they are not necessarily in thermal equilibrium with the surrounding plasma.

3. $\tau_{\nu \text {,tot }} \sim 1$, the semitransparent regime. The solution of the Boltzmann transport problem would be here ideal to model the radiation transport with accuracy. The surfaces defined by the conditions $\tau_{\nu}=2 / 3$ are called neutrino surfaces (or neutrinospheres, in spherically symmetric models). In the case of $\tau_{\nu \text {,tot }}$, they are considered as the last-interaction surfaces, before neutrinos can stream away freely. For $\tau_{\nu, \text { en }}$, they represent the surface at which neutrinos decouple thermally from matter.

4. $\tau_{\nu \text {,tot }} \lesssim 1$, the free-streaming regime. In this regime, neutrinos that are locally produced can stream out freely, almost with no interaction with matter. At the same time, a fraction of the large neutrino fluxes coming from the neutrino surfaces can be here reabsorbed by matter.

\subsection{The Coupling with Hydrodynamics}

In the following we consider a Newtonian hydrodynamical system at time $t$, which is evolving in time with a time step $\Delta t$. To be more general, we consider a 3D domain, and, unless it is explicitly said, no symmetries are assumed. The system is described by its density $\rho$, temperature $T$, electron fraction $Y_{e}$, and velocity field $\boldsymbol{v}$. The trapped neutrino components are defined by $Y_{\nu}$ and $Z_{\nu}$. All these quantities are given at every position $\boldsymbol{x}$. We define the vector of conserved variables, $U$, and 
the corresponding flux tensor, $F$, as

$$
U=\left(\begin{array}{c}
\rho \\
\rho \boldsymbol{v} \\
\rho\left(e+\frac{v^{2}}{2}\right) \\
\rho Y_{\mathrm{e}} \\
\rho Y_{\nu} \\
\left(\rho Z_{\nu}\right)^{\frac{3}{4}}
\end{array}\right), F=\left(\begin{array}{c}
\boldsymbol{v} \rho \\
\boldsymbol{v} \rho \boldsymbol{v}+\mathbb{I} P \\
\boldsymbol{v} \rho\left(e+\frac{v^{2}}{2}+\frac{P}{\rho}\right) \\
\boldsymbol{v} \rho Y_{\mathrm{e}} \\
\boldsymbol{v} \rho Y_{\nu} \\
\boldsymbol{v}\left(\rho Z_{\nu}\right)^{\frac{3}{4}}
\end{array}\right),
$$

where the specific internal energy $e$ and the fluid pressure $P$ are provided by an EOS as functions of $\left(\rho, T, Y_{e}\right)$, while $v$ is the modulus of the fluid velocity. The evolution of the system is determined by

$$
\frac{\partial}{\partial t} U+\nabla \cdot F=g_{\text {grav }}+g_{\nu}
$$

where $g_{\text {grav }}$ is the gravitational source term, depending on the gravitational potential $\phi$, and $g_{\nu}$ is the neutrino source term. The latter is related to the variation of the specific internal energy, $\dot{e}$, of the electron fraction, $\dot{Y}_{e}$, of the neutrino trapped components, $\dot{Y}_{\nu}$ and $\dot{Z}_{\nu}$, and of the fluid velocity, $\dot{\boldsymbol{v}}$, provided by neutrinos:

$$
g_{\text {grav }}=\left(\begin{array}{c}
0 \\
-\rho \nabla \phi \\
-\rho \boldsymbol{v} \cdot \nabla \phi \\
0 \\
0 \\
0
\end{array}\right), g_{\nu}=\left(\begin{array}{c}
0 \\
\rho \dot{\boldsymbol{v}} \\
\rho \dot{\boldsymbol{e}}+\rho \boldsymbol{v} \cdot \dot{\boldsymbol{v}} \\
\rho \dot{Y}_{e} \\
\rho \dot{Y}_{\nu} \\
\frac{3}{4} Z_{\nu} \rho^{3 / 4} \frac{\dot{Z}_{\nu}}{Z_{\nu}}
\end{array}\right)
$$

The goal of the ASL scheme is the estimation of the neutrino source term, $g_{\nu}$, from the present values of the thermodynamical state $U$ of the system. Before proceeding, it is important to notice that any leakage scheme models the local net loss of leptons in the form of neutrinos, i.e., the variations of the total (trapped) lepton number, $\dot{Y}_{1}$, and of the specific total internal energy, $\dot{u}$. These quantities are related to the variation rates appearing in Equation (9) by

$$
\dot{Y}_{1}=\dot{Y}_{e}+\dot{Y}_{\nu_{e}}-\dot{Y}_{\bar{\nu}_{e}}
$$

and

$$
\dot{u}=\dot{e}+\frac{1}{m_{\mathrm{b}}}\left(\dot{Z}_{\nu_{e}}+\dot{Z}_{\bar{\nu}_{e}}+4 \dot{Z}_{\nu_{\mu, \tau}}\right)
$$

Note that the contributions to $\dot{Y}_{1}$ given by $\nu_{\mu, \tau}$ and $\bar{\nu}_{\mu, \tau}$ cancel in our approach. Concerning the neutrino stress, we distinguish $\dot{v}$ into two contributions:

$$
\dot{\boldsymbol{v}}=(\dot{\boldsymbol{v}})_{\tau_{\nu}>1}+(\dot{\boldsymbol{v}})_{\tau_{\nu} \lesssim 1}
$$

i.e., one related to the trapped neutrinos, $(\dot{\boldsymbol{v}})_{\tau_{\nu}>1}$, and one to the absorption of radiation in optically thin conditions, $(\dot{\boldsymbol{v}})_{\tau_{\nu} \lesssim 1}$.

In Section 2.3 we show how we compute the rates for the trapped components $\dot{Y}_{\nu}$ and $\dot{Z}_{\nu}$ and the neutrino stress in trapped conditions $(\dot{\boldsymbol{v}})_{\tau_{\nu}>1}$. The calculation of the leakage rates, $\dot{u}$ and $\dot{Y}_{1}$, and of the neutrino stress in free-streaming conditions, $(\dot{v})_{\tau_{\nu}<1}$, is exposed in Section 2.4. In both sections, we omit the explicit dependence on time and position in the equations, apart from when the neutrino energy is involved. In that case, we only omit the temporal dependence.

\subsection{Trapped Component Rates}

The variation rates for the trapped components are computed as

$$
\begin{gathered}
\dot{Y}_{\nu}=\frac{\tilde{Y}_{\nu, t+\Delta t}-Y_{\nu}}{\Delta t}, \\
\dot{Z}_{\nu}=\frac{\tilde{Z}_{\nu, t+\Delta t}-Z_{\nu}}{\Delta t},
\end{gathered}
$$

where $\tilde{Y}_{\nu, t+\Delta t}$ and $\tilde{Z}_{\nu, t+\Delta t}$ are guesses of the trapped components at $t+\Delta t$, only due to neutrino processes. Their computation is done following these steps: (i) Reconstruct approximated trapped components of the neutrino distribution functions, $f_{\nu}^{\mathrm{tr}}$, at the current time $t$ based on $Y_{\nu}$. This is done assuming that

$$
f_{\nu}^{\mathrm{tr}}(E, \boldsymbol{x})=\gamma(\boldsymbol{x})\left(f_{\nu}(E, \boldsymbol{x})\right)_{\mathrm{eq}}\left(1-e^{-\tau_{\nu, \mathrm{en}}(E, \boldsymbol{x})}\right) .
$$

The exponential cutoff ensures that $f_{\nu}^{\text {tr }}$ is significantly different from 0 only when neutrino trapping conditions are fulfilled with respect to the energy optical depth, i.e., $\tau_{\text {en }} \gtrsim 1$, and that it is proportional to $\left(f^{\mathrm{tr}}\right)_{\mathrm{eq}}$ for $\tau_{\text {en }} \gg 1$. The local parameter $\gamma(\boldsymbol{x})$ is fixed by Equation (5). ${ }^{5}$ The equilibrium distribution functions are assumed to be Fermi-Dirac distribution functions of a neutrino gas in thermal and weak equilibrium with matter

$$
\left(f_{\nu}\right)_{\mathrm{eq}}(E, \boldsymbol{x})=\frac{1}{e^{\left(E / T_{\nu}(\boldsymbol{x})-\eta_{\nu}(\boldsymbol{x})\right)}+1},
$$

where $T_{\nu}$ is assumed to be equal to the matter temperature (true for $\tau_{\nu \text {, en }} \gtrsim 1$ ) and $\eta_{\nu}$ is the neutrino degeneracy parameter. For $\nu_{e}$ and $\bar{\nu}_{e}$, we use the equilibrium degeneracy parameter:

$$
\eta_{\nu_{e}}=\left(\mu_{e}-\mu_{n}+\mu_{p}\right) / T=-\eta_{\bar{\nu}_{e}},
$$

where $\mu_{n}, \mu_{p}$, and $\mu_{e}$ are the relativistic chemical potentials of neutrons, protons, and electrons, respectively. For $T \gtrsim 0.5 \mathrm{MeV}$, we assume $\mu_{e^{+}}+\mu_{e^{-}}=0$. If $\nu_{\mu, \tau}$ are produced by electron-positron annihilation, this relation suggests that neutrinos and antineutrinos of these flavors have approximately opposite chemical potentials. However, the substantial equivalence between $\nu_{\mu, \tau}$ and $\bar{\nu}_{\mu, \tau}{ }^{6}$ implies also the equivalence between their chemical potentials. Thus, the degeneracy parameter for $\nu_{\mu, \tau}$ is set to 0 .

(ii) Evolve $f_{\nu}^{\text {tr }}$ according to timescale arguments between $t$ and $t+\Delta t$. This is done considering the neutrino production and diffusion as two competing processes:

$$
\frac{d f_{\nu}^{\operatorname{tr}}}{d t}=\dot{f}_{\nu, \text { prod }}^{\operatorname{tr}}+\dot{f}_{\nu, \text { diff }}^{\operatorname{tr}},
$$

\footnotetext{
5 We notice that the usage of $Y_{\nu}$ to reconstruct $f_{\nu}^{\text {tr }}$ does not ensure the exact reconstruction of $Z_{\nu}$. On one hand, we have tested that the usage of $Z_{\nu}$ instead of $Y_{\nu}$ does not change our results significantly. On the other hand, the usage of the original (i.e., not reconstructed) values of $Z_{\nu}$ in the computation of $\dot{Z}_{\nu}$ still ensures energy conservation.

6 This approximation is valid as long as the temperature is not high enough to produce a significant amount of muons and antimuons $\left(T \ll m_{\mu} \approx 105.7 \mathrm{MeV}\right.$ ) and nuclear effects distinguishing $\nu$ and $\bar{\nu}$ (like weak magnetism) are neglected.
} 
with

$$
\dot{f}_{\nu, \text { prod }}^{\mathrm{tr}}=\frac{\left(\left(f_{\nu}\right)_{\mathrm{eq}}-f_{\nu}^{\mathrm{tr}}\right)}{\max \left(t_{\nu, \mathrm{prod}}, \Delta t\right)} \exp \left(-\frac{t_{\nu, \mathrm{prod}}}{t_{\nu, \mathrm{diff}}}\right)
$$

and

$$
\dot{f}_{\nu, \text { diff }}^{\mathrm{tr}}=-\frac{f_{\nu}^{\mathrm{tr}}}{\max \left(t_{\nu, \text { diff }}, \Delta t\right)} \exp \left(-\frac{t_{\nu, \text { diff }}}{t_{\nu, \text { prod }}}\right) .
$$

In Equations (19) and (20), all the quantities are evaluated at position $\boldsymbol{x}$ and for a certain neutrino energy $E$. In Equation (19), the term before the exponential ensures that the distribution function reaches the equilibrium value whenever the production timescale is small enough compared to the time step $\Delta t$. Similarly, the first part of Equation (20) causes the distribution function to go to 0 , if the diffusion timescale is small enough compared with $\Delta t$. The exponential factors in both expressions are a switch between the diffusive and the free-streaming regime. The production timescale, $t_{\nu \text {,prod }}$, is set by the neutrino emissivity:

$$
t_{\nu, \operatorname{prod}}(E, \boldsymbol{x})=\frac{1}{j_{\nu}(E, \boldsymbol{x})}
$$

Following Rosswog \& Liebendörfer (2003) (see Ruffert et al. 1996), we define the diffusion timescale, $t_{\nu \text {,diff, as }}$

$$
t_{\nu, \mathrm{diff}}(E, \boldsymbol{x})=\frac{\Delta x_{\nu}(E, \boldsymbol{x})}{c} \tau_{\nu, \text { tot }}(E, \boldsymbol{x})
$$

The quantity $\Delta x_{\nu}$ can be understood as the effective width of a layer drained by the diffusion flux, and it is calculated as

$$
\Delta x_{\nu}(E, \boldsymbol{x})=\alpha_{\text {diff }} \tau_{\nu, \text { tot }}(E, \boldsymbol{x}) \lambda_{\nu, \text { tot }}(E, \boldsymbol{x}) .
$$

Usually, $\alpha_{\text {diff }} \sim 3$ (e.g., Mihalas \& Mihalas 1984; Ruffert et al. 1996 and Rosswog \& Liebendörfer 2003). While for large optical depths Equation (22) provides an estimate of a proper diffusion timescale, in the optically thin regime its value decreases significantly, owing to its quadratic dependence on the optical depth.

(iii) Obtain $\tilde{Y}_{\nu, t+\Delta t}$ and $\tilde{Z}_{\nu, t+\Delta t}$ from $f_{\nu}^{\mathrm{tr}}(t+\Delta t, E, \boldsymbol{x})$, based on Equations (5) and (6).

Trapped neutrinos provide a source of stress for the fluid. This stress is determined by the gradient of the pressure of the neutrino gas

$$
(\dot{\boldsymbol{v}})_{\tau_{\nu}>1}=-\frac{\nabla P_{\nu, \mathrm{tot}}}{\rho}
$$

where the neutrino pressure is evaluated based on the energy content of the neutrino gas,

$$
P_{\nu, \text { tot }}=\sum_{\nu} P_{\nu}=\frac{1}{3} \frac{\rho}{m_{b}}\left(Z_{\nu_{e}}+Z_{\bar{\nu}_{e}}+4 Z_{\nu_{\mu, \tau}}\right) .
$$

\subsection{Emission Rates}

The rates for the total lepton number and total specific internal energy are obtained as the net balance between the emission rates ( $R_{\nu}^{0}$ for the particles and $R_{\nu}^{1}$ for the energy) and the absorption rates in optically thin conditions $\left(H_{\nu}^{0}\right.$ for the particles and $H_{\nu}^{1}$ for the energy):

$$
\dot{Y}_{1}=-m_{b}\left(R_{\nu_{e}}^{0}-R_{\bar{\nu}_{e}}^{0}-H_{\nu_{e}}^{0}+H_{\bar{\nu}_{e}}^{0}\right),
$$

and

$$
\dot{u}=-\left(R_{\nu_{e}}^{1}+R_{\bar{\nu}_{e}}^{1}+4 R_{\nu_{\mu, \tau}}^{1}\right)+\left(H_{\nu_{e}}^{1}+H_{\bar{\nu}_{e}}^{1}\right) .
$$

In Equations (26) and (27), $\nu_{\mu, \tau}$ and $\bar{\nu}_{\mu, \tau}$ do not provide any net contribution to the lepton number and do not contribute to the absorption in optically thin conditions. The emission and absorption rates are obtained from spectral emission $\left(r_{\nu}\right)$ and absorption $\left(h_{\nu}\right)$ rates, according to

$$
\begin{aligned}
& R_{\nu}^{k}(\boldsymbol{x})=\int_{0}^{+\infty} r_{\nu}(E, \boldsymbol{x}) E^{2+k} d E, \\
& H_{\nu}^{k}(\boldsymbol{x})=\int_{0}^{+\infty} h_{\nu}(E, \boldsymbol{x}) E^{2+k} d E,
\end{aligned}
$$

with $k=0,1$.

The emission rates are computed as smooth interpolation between the production rates,

$$
r_{\nu, \operatorname{prod}}(E, \boldsymbol{x})=\frac{4 \pi}{(h c)^{3}} \frac{j_{\nu}(E, \boldsymbol{x})}{\rho(\boldsymbol{x})},
$$

and the diffusion rates,

$$
r_{\nu, \mathrm{diff}}(E, \boldsymbol{x})=\frac{4 \pi}{(h c)^{3}} \frac{1}{\rho(\boldsymbol{x})} \frac{\left(f_{\nu}\right)_{\mathrm{eq}}(E, \boldsymbol{x})}{t_{\nu, \mathrm{diff}}(E, \boldsymbol{x})} .
$$

The former are expected to be dominant in optically thin conditions, while the latter in the opaque region. The interpolation formula is provided by

$$
\begin{aligned}
r_{\nu}(E, \boldsymbol{x})= & \left(1-\alpha_{\nu, \mathrm{blk}}\right) \tilde{r}_{\nu}(E, \boldsymbol{x}) \\
& \times \frac{1}{\Psi_{\nu}(\boldsymbol{x})} \exp \left(-\tau_{\nu, \mathrm{en}}(E, \boldsymbol{x}) / \tau_{\text {cut }}\right),
\end{aligned}
$$

where

$$
\tilde{r}_{\nu}(E, \boldsymbol{x})=\frac{r_{\nu, \operatorname{prod}}(E, \boldsymbol{x}) \times r_{\nu, \mathrm{diff}}(E, \boldsymbol{x})}{r_{\nu, \operatorname{prod}}(E, \boldsymbol{x})+r_{\nu, \mathrm{diff}}(E, \boldsymbol{x})}
$$

is the interpolation expression used for the emission rates in gray leakage schemes (see, e.g., Ruffert et al. 1996; Rosswog \& Liebendörfer 2003). $\alpha_{\nu, \text { blk }}$ and $\tau_{\text {cut }}$ are parameters, and $\Psi_{\nu}$ is a local normalization factor:

$$
\Psi_{\nu}(\boldsymbol{x})=\frac{\int_{0}^{+\infty} \tilde{r}_{\nu}(E, \boldsymbol{x}) e^{-\tau_{\nu, \mathrm{en}}(E, \boldsymbol{x}) / \tau_{\mathrm{cut}}} E^{2} d E}{\int_{0}^{+\infty} \tilde{r}_{\nu}(E, \boldsymbol{x}) E^{2} d E} .
$$

Then, for $\alpha_{\nu \text {,blk }}=0$ and $\tau_{\text {cut }} \rightarrow+\infty$, we obtain $r_{\nu}=\tilde{r}_{\nu}$. Finite, nonzero values of these constants introduce two important improvements in the ASL scheme.

i. In the case where $\alpha_{\mathrm{blk}}=0$, the total amount of emitted $\nu_{e}$ and $\bar{\nu}_{e}$ is usually overestimated. When a large fraction of the emission rates is produced in the semitransparent and optically thin conditions, the interpolation favors the usage of the production rates, calculated as integral over the whole solid angle of isotropic emission rates, Equation (30). However, a significant fraction of those neutrinos are emitted toward the optically thick region. Moreover, the emission rates can be significantly reduced 
by Pauli's blocking factors, provided by the large amount of free-streaming neutrinos emitted at the neutrino surface or locally produced. To efficiently take into account these effects, we have introduced the factor $\left(1-\alpha_{\nu, \text { blk }}\right)$ in Equation (32). $\alpha_{\nu, \mathrm{blk}}$ is a free parameter of the model, and it is expected to be $\sim 0.5$ for $\nu_{e}$ and $\bar{\nu}_{e}$ in strongly accreting systems. On the other hand, since the emission of $\nu_{\mu, \tau}$ from optically thin conditions is usually negligible, we use $\alpha_{\mathrm{blk}, \nu_{\mu, \tau}} \sim 0$.

ii. The rates obtained with Equation (33) retain spectral information of the local thermodynamical properties of matter. Nevertheless, during the diffusion process, highenergy neutrinos coming from optically thick regions thermalize to lower energies, at least as long as $\tau_{\nu, \text { en }} \gg 1$. Thus, the spectrum emerging from the neutrinospheres is softer than the one provided by Equation (33). To mimic this transition, we have included the term $\exp \left(-\tau_{\nu, \text { en }} / \tau_{\text {cut }}\right) / \Psi_{\nu}$ in Equation (32). The definition of $\Psi_{\nu}$, Equation (34), ensures that the number of neutrinos emitted is preserved, while their final spectrum is modeled according to the energy optical depth. Since a few inelastic interactions are necessary to thermalize the spectrum, we expect $\tau_{\text {cut }} \sim \mathcal{O}(10)$.

\subsubsection{Absorption Rates}

Eventually, neutrinos emitted at the neutrino surface and above it stream away in the optically thin region, with a nonnegligible probability to be reabsorbed by the fluid. In the ASL scheme, we include an estimate of this nonlocal absorption rate, $h_{\nu}(E, \boldsymbol{x})$, based on the computation of the neutrino densities outside the neutrino surfaces. The spectral absorption rates for $\nu_{e}$ and $\bar{\nu}_{e}$ in optically thin conditions are calculated as

$$
\begin{aligned}
h_{\nu}(E, \boldsymbol{x})= & \frac{1}{\rho(\boldsymbol{x})} n_{\nu, \tau_{\nu} \lesssim 1}(E, \boldsymbol{x}) \\
& \times \chi_{\nu, \mathrm{ab}}(E, \boldsymbol{x}) \mathcal{F}_{e}(E, \boldsymbol{x}) \mathcal{H}(E, \boldsymbol{x}) .
\end{aligned}
$$

$\mathcal{F}_{e, \nu}$ is the Pauli blocking factor for electrons or positrons in the final state:

$$
\mathcal{F}_{e^{\mp}}=\left(1-\frac{1}{\exp \left(\left(E \pm Q \mp \mu_{e}\right) / T\right)+1}\right)
$$

where we have assumed that the electron or positron produced by the absorption of an electron neutrino or antineutrino, respectively, has an energy equal to the energy of the incoming neutrino, corrected by the mass difference $Q=\left(m_{n}-m_{p}\right) c^{2} \approx 1.293 \mathrm{MeV} . \quad$ In Equation (35), $\mathcal{H}(E, \boldsymbol{x}) \equiv \exp \left(-\tau_{\nu, \text { tot }}(E, \boldsymbol{x})\right)$ is an exponential term that ensures the application of the heating rates only in the optically thin region. The quantity $n_{\nu, \tau_{\nu}} \leq 1$ is the spectral neutrino density, defined such that the total neutrino density outside the neutrino surface, $N_{\nu, \tau_{\nu} \lesssim 1}$, is

$$
N_{\nu, \tau_{\nu} \lesssim 1}(\boldsymbol{x})=\int_{0}^{+\infty} n_{\nu, \tau_{\nu} \lesssim 1}(E, \boldsymbol{x}) E^{2} d E .
$$

One of the limits of any leakage scheme is that it does not model the spatial and angular distribution of the emission outside the neutrino surface. Thus, the calculation of $h_{\nu}$ requires first a separate evaluation of $n_{\nu, \tau_{\nu} \leq 1}$. This task depends strongly on the nature of the problem and on the symmetry of the system. For spherically symmetric models, the preferential propagation direction is the radial one, and the neutrino densities are related to the spherically symmetric neutrino spectral particle luminosities, $l_{\nu}$, by

$$
n_{\nu, \tau_{\nu} \lesssim 1}(E, R)=\frac{l_{\nu}(E, R)}{4 \pi R^{2} c \mu_{\nu}(E, R)}
$$

where $R$ is the radial coordinate (e.g., Janka 2001). This conversion between the neutrino flux and density involves the (spectral) flux factor $\mu_{\nu}(E, R)$. It represents the average of the cosine of the propagation angle for the free-streaming neutrinos. Far from the neutrinospheres, the distribution functions are expected to peak in the forward direction, meaning $\mu_{\nu}\left(R \gg R_{\nu}\right) \sim 1$. Close to the neutrinospheres, assuming that radiation is emitted isotropically above the plane tangential to the neutrinospheres, $\mu_{\nu}\left(R \sim R_{\nu}\right) \sim 1 / 2$. Following Liebendörfer et al. (2009), we use an analytic approximation for $R>R_{\nu}(E)$ :

$$
\mu_{\nu}(E, R)=\frac{1}{2}\left(1+\sqrt{1-\left(\frac{R_{\nu}(E)}{\max \left(R, R_{\nu}(E)\right)}\right)^{2}}\right) .
$$

The quantity $l_{\nu}$ is computed at each radius and for each neutrino energy as a solution of the differential equation

$$
\begin{aligned}
\frac{d l_{\nu}(E, R)}{d R}= & 4 \pi R^{2} \rho(R) r_{\nu}(E, R) \\
& -\frac{\chi_{\mathrm{ab}}(E, R)}{c} \mathcal{H}(E, R) l_{\nu}(E, R) .
\end{aligned}
$$

The neutrinos absorbed in free-streaming conditions deposit momentum in the fluid. In the case of spherically symmetric models, the related stress is computed as

$$
(\dot{\boldsymbol{v}})_{\tau_{\nu} \lesssim 1}(R)=\frac{1}{c} \int_{0}^{+\infty} h(E, R) \mu_{\nu}(E, R) E^{3} d E .
$$

For systems with an approximate spherical symmetry (like collapsing stellar cores), the procedure described above to compute $n_{\nu}$ and $(\dot{\boldsymbol{v}})_{\tau_{\nu} \leq 1}$ can be applied in a ray-by-ray fashion (i.e., along radial paths starting from the center of the system). For more general geometries, ray-tracing algorithms can be designed (e.g., Perego et al. 2014b).

\section{CALIBRATION AND VALIDATIONS}

We implement the ASL scheme, as it is described in Section 2, in the implicit, spherically symmetric hydrodynamics code Agile (e.g., Liebendörfer et al. 2002; Fischer et al. 2010, and references therein). For the calculation of the neutrino emissivities, absorptivities, and scattering rates, we include a minimal set of neutrino-matter reactions containing the most relevant ones. For the production and the absorption of electron flavor neutrinos,

$$
\begin{aligned}
& e^{-}+p \leftrightarrow n+\nu_{e}, \\
& e^{-}+(A, Z) \leftrightarrow(A, Z-1)+\nu_{e}, \\
& e^{+}+n \leftrightarrow p+\bar{\nu}_{e} .
\end{aligned}
$$

where $e^{-}$and $e^{+}$refer to electrons and positrons, while $n, p$, and $(A, Z)$ to neutrons, protons, and nuclei with mass number $A$ and atomic number $Z$, respectively. Pair processes, like electron-positron annihilation and neutrino bremsstrahlung 
from free nucleons (generically referred to as $N$ ), are expected to be secondary sources for $\nu_{e}$ and $\bar{\nu}_{e}$, but primary for $\nu_{\mu, \tau}$ :

$$
\begin{gathered}
e^{-}+e^{+} \leftrightarrow \nu+\bar{\nu}, \\
N+N \leftrightarrow N+N+\nu+\bar{\nu} .
\end{gathered}
$$

All the reactions listed above are considered as inelastic. Major sources of opacity for all neutrino species are provided by scattering on nucleons and nuclei:

$$
\begin{aligned}
N+\nu & \rightarrow N+\nu, \\
(A, Z)+\nu & \rightarrow(A, Z)+\nu .
\end{aligned}
$$

These scattering reactions are considered as elastic in the computation of the total and energy mean free paths. All these weak interactions are implemented according to Bruenn (1985), apart from neutrino bremsstrahlung (Hannestad \& Raffelt 1998) and pair production (Bruenn 1985; Mezzacappa \& Messer 1999), whose implementation is described in Appendix A. The opacity provided by the scattering of neutrinos on electrons and positrons has not yet been implemented. It has been shown that this process is relevant to thermalize neutrinos during the collapse of the core (e.g., Mezzacappa \& Bruenn 1993b) and in the cooling phase of the proto-neutron star (PNS) in exploding models, seconds after core bounce (Fischer et al. 2012). Even if the effect of this reaction on the total and energy mean free path is not considered, the thermalization effect provided by it in the optically thick regime is partially taken into account in our scheme by enforcing the usage of equilibrium Fermi-Dirac distribution functions to model the neutrino trapped component. The impact of this effective treatment in the different parts of a core-collapse simulation is discussed later in more detail.

\section{1. $15 \mathrm{M}_{\odot}$ Progenitor}

The ASL scheme is an effective treatment for neutrinoradiation hydrodynamics. Therefore, it requires being tested and compared against a reference solution, not only to check its validity and accuracy but also to set the free parameters that appear in the scheme. To perform this test, we choose the case of spherically symmetric core-collapse models. We start with a zero-age main-sequence (ZAMS) $15 M_{\odot}$ progenitor model, obtained by Woosley et al. (2002). We follow the collapse of the core and the first $300 \mathrm{~ms}$ after core bounce. We include $\approx 2.05 M_{\odot}$ from the initial progenitor, distributed over 103 radial zones on the adaptive Lagrangian grid of Agile. The corresponding initial outer radius is $\approx 7500 \mathrm{~km}$ from the origin. As a reference solution, we use the results obtained by the BOLTZTRAN code (Mezzacappa \& Bruenn 1993a, 1993b, 1993c; Mezzacappa \& Messer 1999; Liebendörfer et al. 2004; Fischer et al. 2012, and references therein), also coupled with the Agile code. BOLTZTRAN solves the Boltzmann equation using the method of discrete ordinates with a Gauss-Legendre quadrature. BOLTZTRAN incorporates all the neutrino reactions listed in Equations (42)-(48). For consistency with our ASL implementation, we rely on the neutrino reaction implementation reported in Liebendörfer et al. (2005). Recently, Lentz et al. (2012a) have shown the impact of modern neutrino rates in Agile-BOLTZTRAN runs. We postpone the implementation of more accurate reaction rates in ASL to a future step. Furthermore, we notice that, even if neutrino-electron scattering (NES) is implemented in the BOLTZTRAN version we are
Table 1

Values of the Parameters Used in the Agile-ASL Runs

\begin{tabular}{lccc}
\hline \hline Name & $\alpha_{\text {diff }}$ & $\alpha_{\text {blk }}$ & $\tau_{\text {cut }}$ \\
\hline \multicolumn{5}{c}{ Calibration Sets } \\
\hline CAL & $\{3,4,5,6\}$ & $\{0.4,0.45,0.5,0.55,0.6\}$ & $\{10,15,20,25\}$ \\
\hline \multicolumn{4}{c}{ Standard Set } \\
\hline STD & $3+2 X_{h}$ & 0.55 & 20 \\
\hline & & Parameter Variation Study & 20 \\
\hline AD_2 & 2 & 0.55 & 20 \\
AD_5 & 5 & 0.55 & 20 \\
AB_45 & $3+2 X_{h}$ & 0.45 & 7 \\
AB_65 & $3+2 X_{h}$ & 0.65 & 54 \\
TC_7 & $3+2 X_{h}$ & 0.55 & 20 \\
TC_54 & $3+2 X_{h}$ & 0.55 & \\
\hline
\end{tabular}

Note. Different sets of free parameters of the model are used in this study. In the calibration runs (CAL), the three parameters are varied independently, producing 80 different parameter sets. The result of the calibration is denoted as standard set (STD) and is also used in the multidimensional runs. The six parameter sets used to show the sensitivity of our models to variations of a single parameter from the STD set are listed as a parameter variation study.

using, for consistency our reference runs do not include it, if not stated otherwise. We perform our tests and compute our reference solutions assuming Newtonian gravity to be able to compare later with different multidimensional Newtonian hydrodynamical schemes. In all our Agile-ASL runs, as well as in the Agile-BOLTZTRAN ones, we use the Lattimer-Swesty EOS (Lattimer \& Swesty 1991), with nuclear compressibility $K=220 \mathrm{MeV}$. Neutrino energies are discretized by 20 geometrically increasing energy groups in the range $3 \mathrm{MeV} \leqslant E_{\nu} \leqslant 300 \mathrm{MeV}$. In the Agile-BOLtzTRAN runs, the neutrino propagation angle is discretized by 6 angular bins.

\subsubsection{Parameter Choice}

The validation and the calibration of the ASL scheme are done by comparing directly the temporal and the radial profiles of some relevant quantities obtained with Agile-ASL with our reference solutions. We do not define a quantitative criterion to compare the different results, because our simulations span a broad range of conditions and this prevents the possibility of selecting a single quantity as an indicator. Instead, we search for the parameter set that, overall, best matches the most important features of a CCSN model between collapse and a few hundreds of milliseconds after core bounce. In particular, during the collapse, we focus on central quantities (e.g., density, entropy, and electron fraction). Before and after bounce, we monitor the profiles of matter density, $\rho$, matter entropy per baryon, $s$, electron fraction, $Y_{e}$, and radial velocity, $v_{r}$. Regarding the neutrino quantities, we investigate the radial profile of $Y_{\nu}$, together with the luminosities, $L_{\nu}$, and the rms neutrino energies, $E_{\mathrm{rms}}=\sqrt{\left\langle E_{\nu}^{2}\right\rangle}$, both measured at $300 \mathrm{~km}$ from the center. The different versions of Agile-ASL differ by the usage of a distinct set of parameters, $\left(\alpha_{\mathrm{diff}}, \alpha_{\mathrm{blk}}\right.$, $\left.\tau_{\text {cut }}\right)$. The ranges we have explored are reported in the first row of Table 1. 

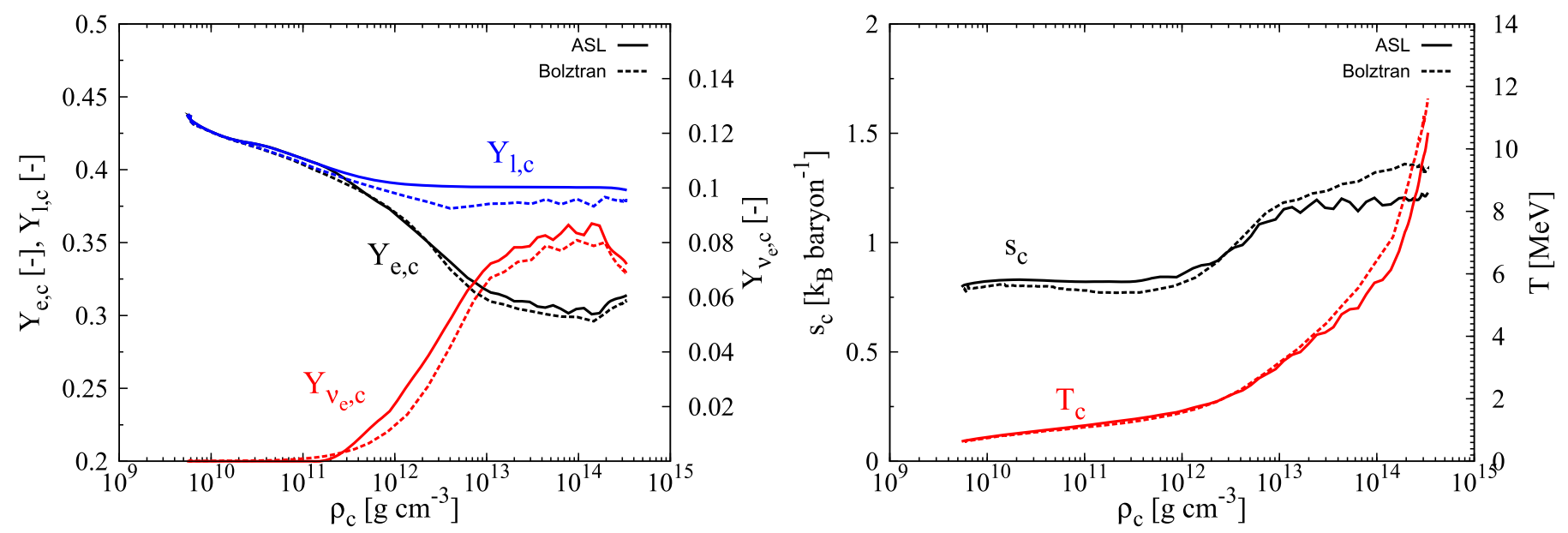

Figure 1. Left: central electron fraction (black lines), electron neutrino fraction (red lines), and lepton fraction (blue lines) evolution during the collapse of the $15 M_{\odot}$ model, as a function of the central density. The solid lines refer to the run obtained with the ASL scheme, while the dashed lines refer to the run obtained with BOLTZTRAN. Right: same as for the left panel, but for central entropy (black lines) and temperature (red lines), as a function of the central density during the collapse.
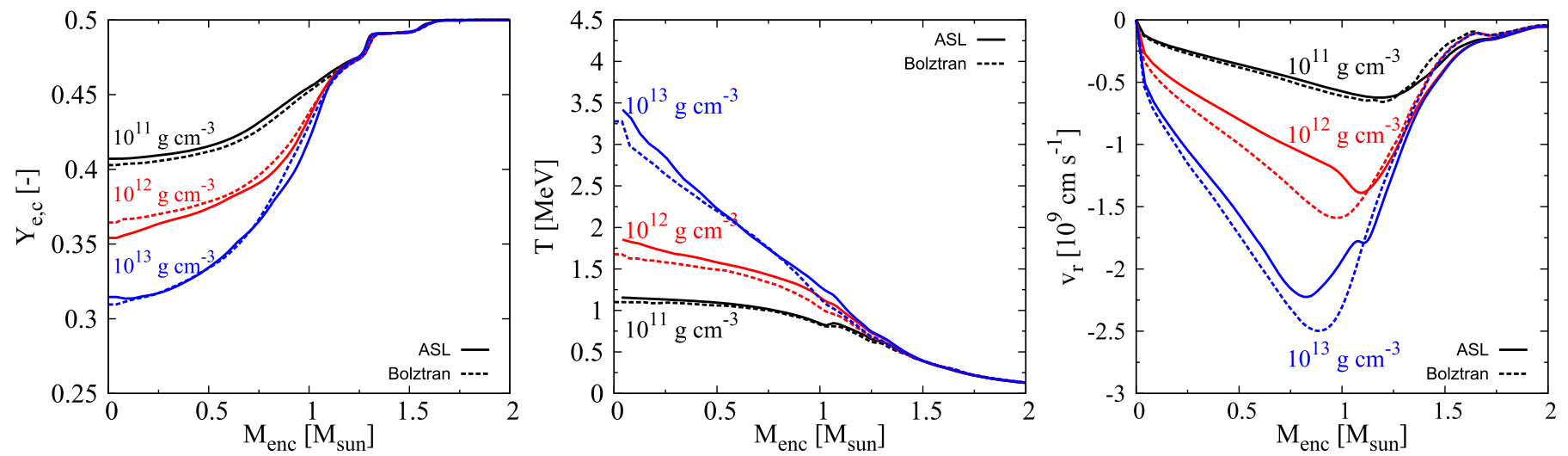

Figure 2. Profiles of the electron fractions (left), temperature (middle), and radial velocity (right) as a function of the enclosed mass profile for the $15 M_{\odot}$ model, at three different times labeled by their central densities (black: $10^{11} \mathrm{~g} \mathrm{~cm}^{-3}$, red: $10^{12} \mathrm{~g} \mathrm{~cm}^{-3}$; blue: $10^{13} \mathrm{~g} \mathrm{~cm}^{-3}$ ). The solid lines represent the run obtained with the ASL scheme, while the dashed lines represent the run obtained with BOLTZTRAN.

The values of the parameters that provide the overall best agreement in all the monitored quantities, during the entire simulation, are as follows.

$\alpha_{\text {diff }}=3\left(1+2 X_{h} / 3\right)$, where $X_{h}$ is the mass fraction of heavy nuclei. This peculiar dependence is a simple interpolation between two limiting behaviors: $\alpha_{\text {diff }} \approx 5$ in the unshocked regions (more relevant in the collapse phase) and $\alpha_{\text {diff }} \approx 3$ in the shocked ones (more relevant in the postbounce phase). The physical interpretation of this difference is linked with the fact that in the unshocked region $\nu_{e}$ and $\bar{\nu}_{e}$ opacities depend more on quasi-elastic scattering on nucleons and nuclei than on inelastic absorption processes $\left(\tau_{\nu \text {,en }} \ll \tau_{\nu \text {,tot }}\right)$, while in the shocked region both are equally important $\left(\tau_{\nu, \text { en }} \lesssim \tau_{\nu \text {,tot }}\right)$. When isoenergetic scattering dominates, high-energy neutrinos diffuse, changing more slowly their energy and interacting more with matter compared with thermalized neutrinos. This effect leads to a significant increase of the diffusion timescale.

$\alpha_{\nu_{e}, \bar{\nu}_{e} \mathrm{blk}}=\alpha_{\mathrm{blk}}=0.55$, while $\alpha_{\nu_{\mu, \tau}, \mathrm{blk}}=0$.

$\tau_{\text {cut }}=20$.

We will refer to this set of values as our standard set (second row in Table 1).

\subsubsection{Collapse and Bounce Phase}

In the following, we describe the results we obtain for our $15 M_{\odot}$ model and how they compare with our reference solution. A comparison of the performances of Agile-ASL and Agile-BOLTZTRAN for the same calibration runs is reported in Appendix B.

The stellar iron core collapses until densities in excess of nuclear saturation density are reached in the center and a shock wave forms. Free-streaming neutrinos reduce the total lepton number, while the electron fraction is further decreased by the conversion of electrons into $\nu_{e}$. The core reaches core bounce on a time $t_{\text {bounce }}=248 \mathrm{~ms}$ after the beginning of the simulation. The central density at bounce is $\rho_{\text {bounce }}=3.36 \times 10^{14} \mathrm{~g} \mathrm{~cm}^{-3}$, and the enclosed mass at the shock formation point is $M_{\text {enc,bounce }}=0.75 M_{\odot}$. The central density and the initial PNS mass compare closely with the corresponding values obtained by Agile-BOLTZTRAN ( $\rho_{\text {bounce, }}$ $\mathrm{AB}=3.34 \times 10^{14} \mathrm{~g} \mathrm{~cm}^{-3}$ and $M_{\text {enc,bounce, } \mathrm{AB}}=0.74 M_{\odot}$, respectively), while the collapse time of the reference model is shorter, $t_{\mathrm{bounce}, \mathrm{AB}}=205 \mathrm{~ms}$. In Figure 1, we compare the central values obtained for $Y_{e}$ and $Y_{\nu_{e}}$ (left panel), and for the entropy and temperature (right panel), as a function of the central density during the collapse. Moreover, in Figure 2, we 

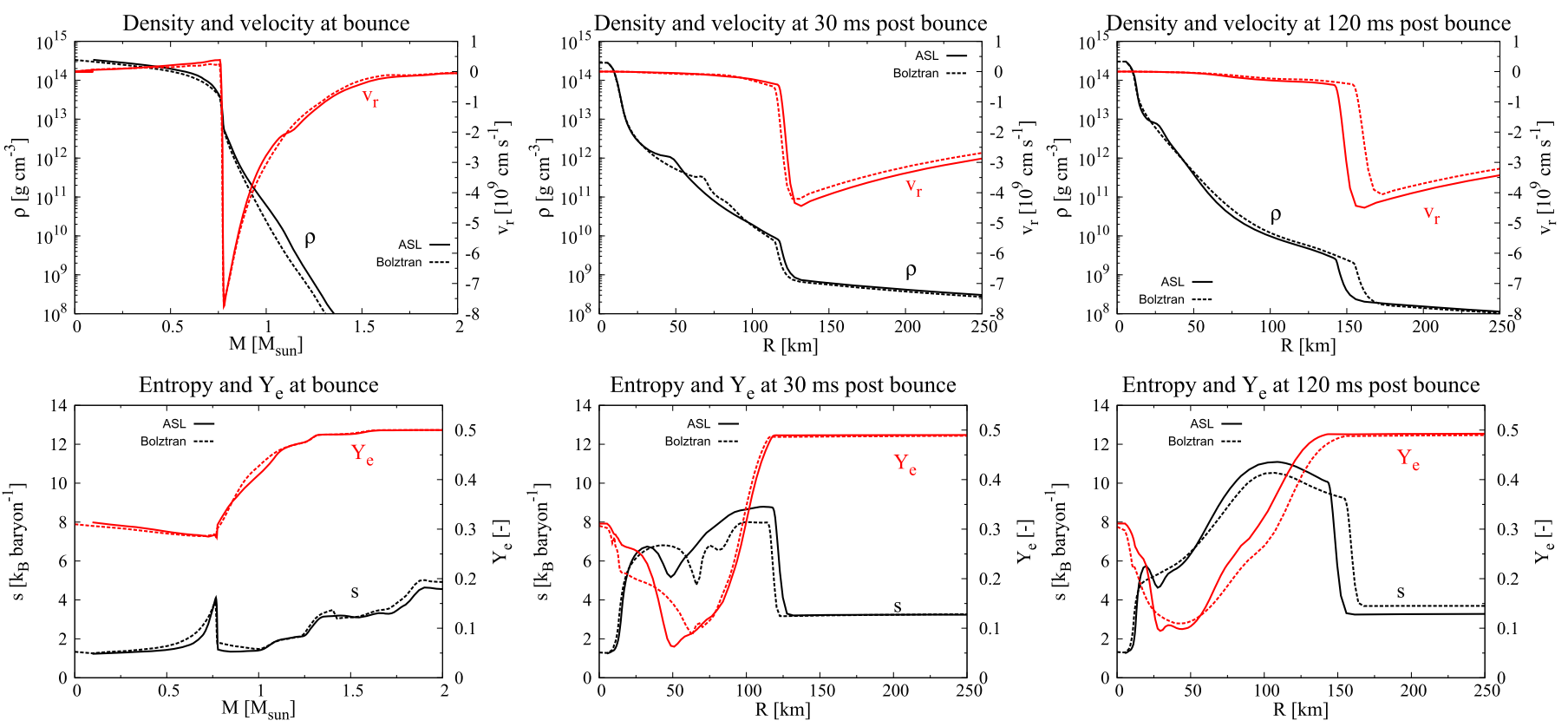

Figure 3. Profiles of the density and radial velocity (top row) and of the entropy and electron fraction (bottom row), at three different times for the $15 M_{\odot}$ model: at core bounce (left panels, using the enclosed mass as an independent variable) and at $30 \mathrm{~ms}$ and $120 \mathrm{~ms}$ after core bounce (middle and right panels, respectively, using the radial distance as an independent variable). The solid lines represent the run obtained with the ASL scheme, while the dashed lines represent the run obtained with BOLTZTRAN.

plot radial profiles of $Y_{e}$, temperature, and radial velocity, as a function of the enclosed mass, for three different times during the collapse (labeled by their central density). In all cases, we have obtained a good agreement with the reference solution. The decrease of $Y_{e}$ is well reproduced during the deleptonization process, while neutrino trapping occurs when the central density reaches $\rho_{\mathrm{c}} \approx 2 \times 10^{12} \mathrm{~g} \mathrm{~cm}^{-3}$. After that, the further decrease of $Y_{e}$ is compensated by the growth in $Y_{\nu_{e}}$, keeping the total lepton number and the entropy roughly constant. In the ASL model, the entropy per baryon and the electron fraction stay almost constant after neutrino trapping up to core bounce, inside the innermost $0.8 M_{\odot}$. In the BOLTZTRAN reference solution, the detailed treatment of the equilibrium approach and diffusion process slightly reduces the total lepton number ( $\Delta Y_{1} \approx 0.02$ ), compared with the ASL solution. Similarly, the entropy per baryon rises just before the formation of the shock wave $\left(\Delta s \approx 0.2 k_{\mathrm{B}}\right)$. This difference is due to full thermal and weak equilibrium with matter assumed in the ASL treatment. However, this equilibrium is only approximated, and deviations from it lead to a slightly larger matter entropy (Cooperstein et al. 1986, 1987). Finally, in the radial velocity profile, we notice a wiggle appearing after neutrino trapping sets in, around $M_{\mathrm{enc}} \approx 1 M_{\odot}$. This is due to the neutrino stress, computed according to Equation (24), which can overestimate the stress at the interface between the trapped and the freestreaming regime and neglects additional momentum transfer in optically thin conditions.

\subsubsection{Post-bounce Phase}

After core bounce, the shock wave propagates outward and iron group nuclei falling into the shock are photodissociated into neutrons and protons. Once the shock reaches the relevant neutrinospheres, electron capture on free protons in almost free-streaming conditions causes a peak in $\nu_{e}$ luminosity and a fast neutronization of the shocked matter. Later, the absorption of electrons and positrons on free nucleons, together with neutrino pair processes, produces an intense radiation emission of neutrinos of all flavors. The combined effect of the nuclei photodissociation and neutrino emission causes the prompt shock expansion to stop and the shock itself to stall within a few tens of milliseconds. The absorption of neutrinos inside the so-called gain region increases the shock radius during several tens of milliseconds after the stalling. However, this energy deposition is not enough to revive the shock and lead to an explosion of the star.

Figure 3 shows radial profiles of several quantities at core bounce, as a function of the enclosed mass, as well as at two different times after core bounce $(t \approx 30 \mathrm{~ms}$ and $t \approx 120 \mathrm{~ms})$, as a function of the radial distance from the center. The results obtained with the ASL scheme show the most relevant features and the expected typical evolution. We find a good agreement for the location of the shock during the different phases. We recognize the effect of the passage of the shock wave in the electron fraction profile, as well as the result of the neutrino emission and absorption on the entropy profile (especially around $120 \mathrm{~ms}$, where the increase of the entropy inside the gain region can be seen). A detailed comparison with the reference solution shows several quantitative differences between the two models. They originate from our approximate treatment, compared to a detailed neutrino transport scheme. However, the overall qualitative (and also a partial quantitative) agreement between the two models is preserved during the entire simulation time.

In the left panel of Figure 4, we present the temporal evolution of the shock and PNS radii (defined as $\rho$ $\left.\left(R_{\mathrm{PNS}}\right)=10^{11} \mathrm{~g} \mathrm{~cm}^{-3}\right)$. Overall, $R_{\text {shock }}$ and $R_{\mathrm{PNS}}$ evolutions are in good agreement with the reference model. In the AgileASL run, the shock expansion reaches its maximum, $\approx 145 \mathrm{~km}$, around $140 \mathrm{~ms}$. This maximum extension is $\sim 10 \%$ smaller than the maximum $R_{\text {shock }}$ obtained by the AgileBOLTZTRAN simulation. We also notice that the latter reaches 

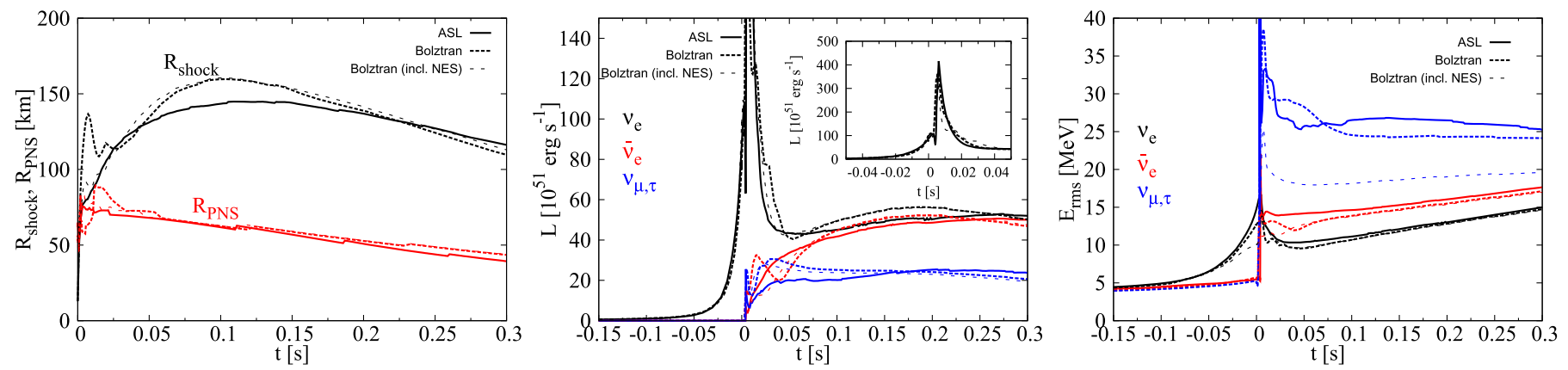

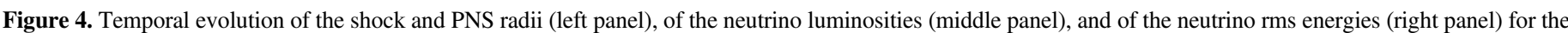

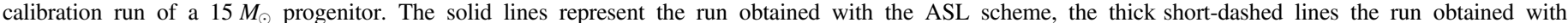
BOLTZTRAN. For comparison purposes, we also plot results obtained with BOLTZTRAN including NES (thin long-dashed lines).

its maximum earlier ( $\approx 105 \mathrm{~ms})$. In general, the shock evolution is more pessimistic in the ASL model than in the reference one. On one hand, this is a consequence of the smaller PNS radius (e.g., Marek \& Janka 2009), which in turn is due to an overestimated neutrino cooling happening in the semitransparent regime. On the other hand, the comparison with a BOLTZTRAN model including also NES (thin long-dashed lines) suggests that the smaller shock radius obtained by the ASL scheme during the first $30 \mathrm{~ms}$ after core bounce could also be the result of the effective inclusion of the neutrino thermalization provided by this inelastic process. This effect, together with the enhanced $\nu_{e}$ luminosity around neutrino burst, compensates for the larger enclosed mass at core bounce. Finally, despite the slightly smaller extension, the PNS contraction rate within the first $300 \mathrm{~ms}$ is similar to the one of the reference solution.

\subsubsection{Neutrino Quantities}

The neutrino luminosities and rms energies are displayed in the middle and right panels of Figure 4.

During the collapse phase, electron capture on nuclei causes the $\nu_{e}$ luminosity to rise. This increase proceeds monotonically up to neutrino trapping $\left(L_{\nu_{e}} \approx 10.6 \times 10^{52} \mathrm{erg} \mathrm{s}^{-1}\right)$, when the enhanced core opacity and the fast-decreasing collapse timescale halt (and even slightly decrease) the $\nu_{e}$ luminosity. Once the core has bounced and the shock has passed through the relevant neutrinospheres, $L_{\nu_{e}}$ shows a burst and peaks at $4.1 \times 10^{53} \mathrm{erg} \mathrm{s}^{-1}$. The same behavior appears in the reference solution obtained by Agile-BOLTZTRAN, even if the luminosities are a bit smaller $\left(9.5 \times 10^{52} \mathrm{erg} \mathrm{s}^{-1}\right.$ at trapping and $3.5 \times 10^{53} \mathrm{erg} \mathrm{s}^{-1}$ at burst). After the neutrino burst, $L_{\nu_{e}}$ decreases and stabilizes during the accretion phase, $L_{\nu_{e}} \approx 5.0 \times 10^{52} \mathrm{erg} \mathrm{s}^{-1}$. At the same time, the $\bar{\nu}_{e}$ luminosity rises and becomes almost equal to $L_{\nu_{e}}$ during the whole accretion phase. The same trend is observed in the reference solution, and the differences in the absolute values are usually within $10 \%$. The smaller values obtained by the ASL scheme are related to the smaller neutrinosphere radii. In correspondence with the sudden $(t \lesssim 25 \mathrm{~ms})$ expansion and contraction of the shock wave observed in the BOLTZTRAN run without NES, the $\bar{\nu}_{e}$ luminosity presents a pronounced oscillation. This increased luminosity is due to the larger presence of hot matter $\left(T \sim\right.$ few MeV) at relatively low density $\left(\rho \gtrsim 10^{11} \mathrm{~g} \mathrm{~cm}^{-3}\right)$ above the PNS. In the BOLTZTRAN runs, these features disappear once NES is included, as a consequence of the more efficient neutrino thermalization and the larger $\nu_{e}$ peak luminosities (see also Figures 5 and 6 in Lentz et al. (2012a), even though there the effects are less evident, owing to the different progenitor structure and potentially to the different opacities used). Also in this case, the results obtained with the ASL scheme follow more closely the BOLTZTRAN run with NES. The rise of $L_{\nu_{\mu, \tau}}$ proceeds as well after core bounce, but a few milliseconds before $L_{\bar{\nu}_{e}}$. This is expected because of the negative $\bar{\nu}_{e}$ degeneracy parameter and the larger opacity that characterize electron antineutrinos compared with $\mu$ and $\tau$ neutrinos. In the rising phase (i.e., within the first $\sim 75 \mathrm{~ms}$ ), the ASL scheme underestimates the $\nu_{\mu, \tau}$ luminosities, while the agreement increases during the stationary accretion phase. This discrepancy is due to the difficulty by the ASL scheme in modeling the dynamical rise of the $\nu_{\mu, \tau}$, which are characterized by an extended scattering atmosphere above the radius where neutrino bremsstrahlung and pair production freeze out.

The rms energies obtained by the ASL scheme show trends in agreement with the reference solution. A harder spectrum is obtained for $\nu_{e}$ during the collapse phase and for $\bar{\nu}_{e}$ in the first tens of milliseconds after core bounce. We notice that the rms energies for $\nu_{\mu, \tau}$ are consistent with the values obtained by the reference solution, i.e., without including NES. However, the inclusion of this process in Agile-BOLTZTRAN leads to significantly smaller energies (by $\sim 20 \%-25 \%$ ). This indicates that this process is responsible for efficiently down-scattering high-energy $\nu_{\mu, \tau}$, while they diffuse out from the core. The same behavior is visible for $\nu_{e}$ during the latest phases of the collapse.

\subsubsection{Parameter Variations}

We briefly explore the sensitivity of the ASL scheme with respect to variations of its free parameters $\left(\alpha_{\text {diff }}, \alpha_{\text {blk }}, \tau_{\text {cut }}\right)$ around the calibrated values. To do this, we consider the $15 M_{\odot}$ model of Section 3.1, with its standard set of parameters, and we vary each of them independently. We choose $\alpha_{\text {diff }}=2$ $\left(\mathrm{AD} \_2\right.$ model $)$ and $\alpha_{\text {diff }}=5$ (AD_5 model), $\alpha_{\text {blk }}=0.45$ (AB_45 model) and $\alpha_{\text {blk }}=0.65$ (AB_65 model), and $\tau_{\text {cut }}=7$ (TC_7 model) and $\tau_{\text {cut }}=54$ (TC_54 model), representing six independent tests (see Table 1). We notice that the diffusion rates are proportional to $1 / \alpha_{\mathrm{diff}}$, and the chosen diffusion parameters could also be expressed as $1 / \alpha_{\text {diff }}=0.5$ (AD_2 model) and $1 / \alpha_{\text {diff }}=0.2$ (AD_5 model). Thus, the variations of $\alpha_{\text {diff }}$ and $\alpha_{\text {blk }}$ span an effective interval of roughly $\pm 30 \%$ around the calibrated values. For $\tau_{\text {cut }}$, we choose two values such that $\ln \left(\tau_{\text {cut }}\right) / \ln (20) \approx 1 \pm(1 / 3)$.

In Figure 5, we show the shock and PNS radius, the $\nu_{e}$ luminosity, and the $\nu_{e}$ rms energy (the corresponding curves for 

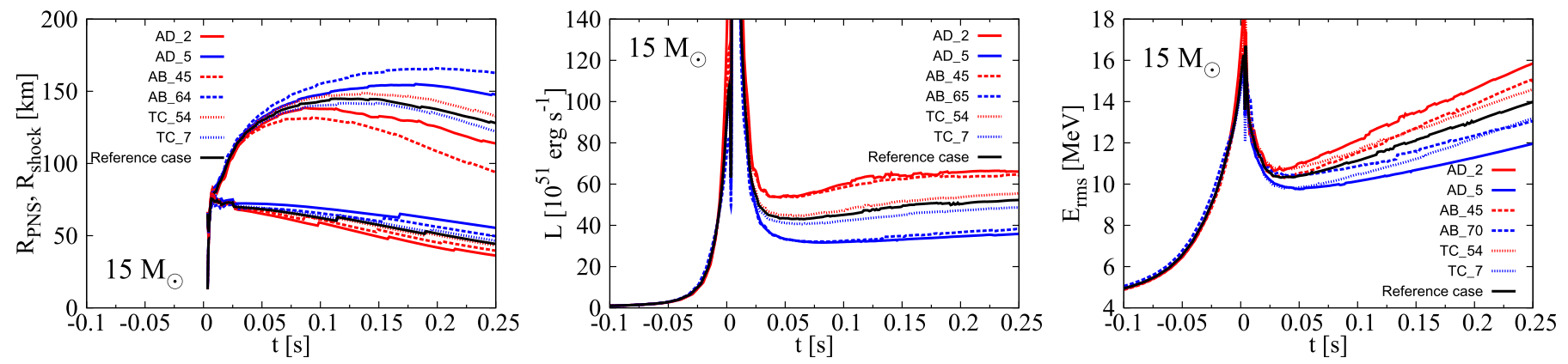

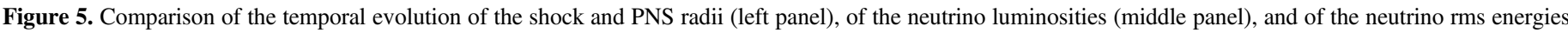
(right panel) between our reference case and six runs obtained varying the three free parameters, $\alpha_{\text {blk }}, \alpha_{\text {diff }}$, and $\tau_{\text {cut }}$ (see the text for details).

$\bar{\nu}_{e}$ and $\nu_{\mu, \tau}$ present analogous trends) for each of the six tests, in comparison with the reference case. The parameter $\alpha_{\text {blk }}$ alters the emission rates everywhere inside the core (see Equation (32)), causing a variation of the total neutrino luminosity roughly equal to the variation of the parameter itself. Since it applies equally to all neutrino energies, it does not affect directly the neutrino spectrum and it modifies only marginally the evolution of the neutrino mean energies. The variations of the rms energies that we observe in AB_45 and AB_65 are mainly due to the different evolution of the radial profiles of the thermodynamical quantities. A variation of $\pm 30 \%$ of $\alpha_{\mathrm{blk}}$ does not change the qualitative behavior of the simulations, but it changes the shock radius significantly, by a few tens of kilometers. Variations of the PNS radius are more restrained.

Since the diffusion rates affect mainly the behavior of the deep interior of the collapsing core, the radius of the PNS is more sensitively affected by variations of the diffusion parameter $\alpha_{\text {diff. }}$. In particular, an increase of $\alpha_{\text {diff }}\left(\mathrm{AD} \_5\right)$ causes a decrease of the diffusion rates and of the neutrino luminosities. At the same time, it slows the PNS contraction down and moves outward the transition region between the diffusion and the production rates (see Equation (33)). The smaller temperature at the PNS surface produces softer neutrino spectra. The shock radius position is determined by the hydrostatic equilibrium configuration inside the hot PNS mantle, under the influence of neutrino cooling and heating (e.g., Janka 2001). The lower neutrino energy deposition is compensated by the more extended PNS, and the net result is a larger shock radius (see Equation (2) of Janka [2012] for the scaling relationships between the shock and PNS radii, the neutrino luminosities, and mean energies). A decrease of $\alpha_{\text {diff }}($ AD_2) provides parallel but opposite effects.

The variations of the parameter $\tau_{\text {cut }}$ have the smallest impact on the ASL scheme results. In particular, a large increase of $\tau_{\text {cut }}$ from 20 to 54 (TC_54) provides larger neutrino mean energies, but it also increases the emission rates associated with highenergy neutrinos, Equation (32). The more intense energy emission coming from the optically thick region is still not enough to modify significantly the evolution of the PNS radius. However, it almost compensates the more efficient absorption provided in optically thin conditions, and it limits the differences in the radial profiles and in the shock conditions. Analogous, but opposite, considerations apply to the TC_7 run.

Our brief parametric study has shown that even significant variations of the free parameters of the model (of the order of $\pm 30 \%$ ) around the calibrated values do not change qualitatively the results of the simulations for the tested $15 M_{\odot}$ progenitor. On the other hand, quantitative differences are present: variations of the parameters $\alpha_{\mathrm{blk}}$ and $\alpha_{\mathrm{diff}}$ have the largest impact, since they modify significantly the neutrino luminosities and mean energies. The diffusion parameter regulates also the contraction rate of the PNS, while it has a less pronounced effect on the neutrino mean energies, compared with $\alpha_{\text {blk }}$. Variations of $\tau_{\text {cut }}$ affect mainly the neutrino mean energies, but have a reduced impact on the overall dynamics.

\section{2. $12 \mathrm{M}_{\odot}$ and $40 \mathrm{M}_{\odot}$ Progenitors}

After having presented the calibration of the ASL free parameters for the core collapse of a $15 M_{\odot}$ ZAMS star, we test them with two different progenitors: $12 M_{\odot}$ (Woosley et al. 2002) and $40 M_{\odot}$ (Woosley \& Heger 2007) ZAMS stars. Also for these cases, we compare the results obtained in Newtonian simulations performed with Agile-ASL with the ones of Agile-BOLTZTRAN. For the $12 M_{\odot}$ case, we include $1.67 M_{\odot}$ from the initial progenitor, distributed over 103 radial zones. The initial radius extends up to $6800 \mathrm{~km}$. For the $40 M_{\odot}$ case, we include $2.60 M_{\odot}$ from the initial progenitor, distributed over 135 radial zones, ranging initially from 0 up to $5100 \mathrm{~km}$ from the center.

The comparison of the collapse profiles between the AgileASL and the Agile-BOLTZTRAN results shows a very good agreement, for example, in the evolution of the central entropy and electron fraction, similar to the one we have observed for the $15 M_{\odot}$ calibration model. The time necessary to reach core bounce is again larger by $15 \%-20 \%$ in the ASL case: 169 and $354 \mathrm{~ms}$, to be compared with 142 and $313 \mathrm{~ms}$ obtained in the detailed neutrino transport run, for the $12 M_{\odot}$ and $40 M_{\odot}$ case, respectively. The enclosed mass where the shock forms is larger in the ASL models by only a few percent (2\%-3\%). In Figure 6, we present the evolution of some key quantities during $250 \mathrm{~ms}$ after core bounce, for both the $12 M_{\odot}$ (top panels) and the $40 M_{\odot}$ (bottom panels) progenitor models. For comparison purposes, we plot also the results obtained by the Agile-BOLTZTRAN code. Overall, the results obtained with the ASL model reproduce qualitatively, and partially quantitatively, the results obtained by the detailed Boltzmann neutrino transport. The agreement is better for the $40 M_{\odot}$ case. This is due to the fact that, despite the large difference in the ZAMS mass, the core properties of the $15 M_{\odot}$ progenitor show similarities with more massive progenitors and differences with lighter progenitors. In the $12 M_{\odot}$ case, the results obtained by the ASL scheme look more pessimistic, owing to a faster and more intense energy loss above the neutrinospheres during the first tens of milliseconds after core bounce. The lower $\nu_{e}$ and $\bar{\nu}_{e}$ luminosities and the larger rms energies, observed for $t \gtrsim 0.75 \mathrm{~ms}$, are a consequence of the more compact PNS 

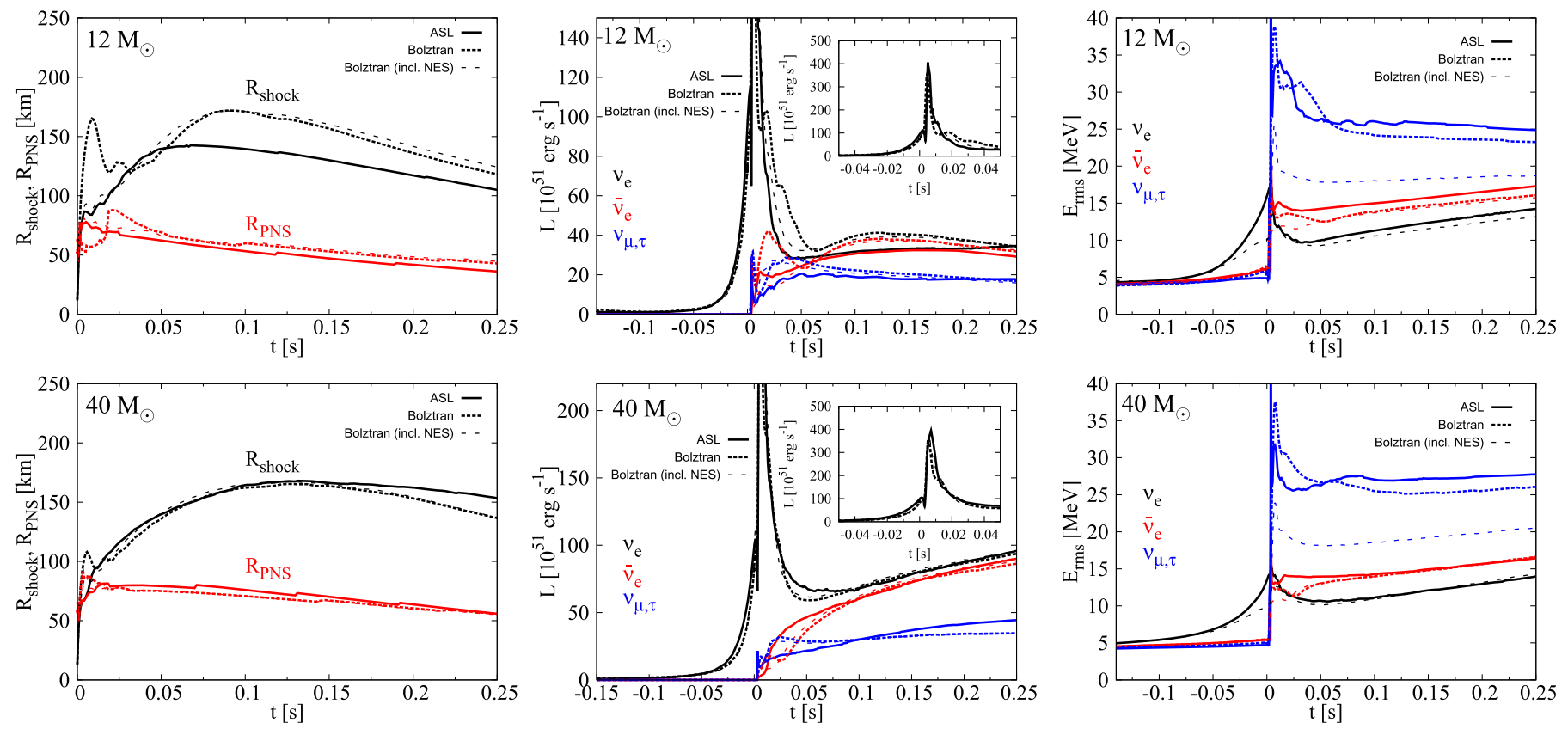

Figure 6. Same as in Figure 4, but for $12 M_{\odot}$ (top panels) and $40 M_{\odot}$ (bottom panels) progenitors.

and shock. Also for these two progenitor models (and especially for the $12 M_{\odot}$ one), the $\nu_{e}$ luminosity and shock radius evolutions within the first tens of milliseconds after core bounce follow more closely the results obtained with BOLTZTRAN, once the neutrino scattering on electrons and positrons has been included. The reasons are analog to the $15 M_{\odot}$ case. In the $12 M_{\odot}$ case, the oscillations in the shock radius and in the $\bar{\nu}_{e}$ luminosities appearing in the BOLTZTRAN runs without NES are even more pronounced than in the $15 M_{\odot}$, while they are practically absent in the $40 M_{\odot}$ case. This is due to the different density structures and accretion rate histories. According to the analysis reported in Section 3.1.5, the more pessimistic results obtained for the $12 M_{\odot}$ case can also indicate that light progenitors would require a slightly different parameter choice to better match results from the reference model (in particular, larger $\alpha_{\text {diff }}$ and $\alpha_{\text {blk }}$ ).

\section{EXAMPLES IN MULTIDIMENSIONAL SIMULATIONS}

In Section 3, we have compared results from the ASL scheme against Boltzmann transport in spherically symmetric models. To do that, we used the same hydrodynamics code, Agile. In order to show the possibility for the scheme to be implemented in multidimensional contexts, we report the following two tests, performed with two different hydrodynamical codes. In the first one, we apply the ASL scheme in a multidimensional setting by coupling it to an axisymmetric Eulerian and nonrelativistic hydrodynamics solver, to model the core collapse of a stellar iron core. In the second one, we couple our algorithm with a Lagrangian hydrodynamics code, and we simulate the same stellar core collapse in 3D using SPH. In both cases we consider a $15 M_{\odot}$ progenitor from Woosley et al. (2002). We use the Lattimer \& Swesty (1991) EOS with nuclear compressibility $K=220 \mathrm{MeV}$ and the ASL standard parameter set, as described in Section 3, with 20 geometrically increasing energy bins between 3 and $300 \mathrm{MeV}$.

The different dimensions, implementations, and numerical techniques are expected to introduce differences among the multidimensional tests and compared with 1D results (see Section 3). Nevertheless, during the collapse phase and in the first tens of milliseconds after core bounce, the profiles and the shock shape are expected to behave similarly to spherically symmetric models (e.g., Marek \& Janka 2009; Müller et al. 2012b; Bruenn et al. 2013), even if deviations due to PNS and prompt convection can appear (e.g., Buras et al. 2006a; Müller et al. 2012b). At later times, multidimensional effects change significantly the dynamics of the system. Several multidimensional CCSN results, employing more sophisticated neutrino treatments, have been published. In the case of axisymmetric models of a $15 M_{\odot}$ model, see, e.g., Buras et al. (2006b), Scheck et al. (2006), Müller et al. (2012a), Bruenn et al. (2013, 2016), Suwa et al. (2013), Zhang et al. (2013), Müller \& Janka (2014), Dolence et al. (2015), and Pan et al. (2015). For 3D SPH models, see, e.g., Fryer \& Warren (2004). They provide reference cases to check the qualitative behavior of our simulations and of the neutrino quantities within a few hundreds of milliseconds after core bounce. However, our focus is to demonstrate the versatility and portability of the ASL algorithm, along with the validation of the results it provides with $1 \mathrm{D}$ detailed simulations, but not to compare to other hydrodynamical codes and among different dimensions.

\subsection{D Grid-CCSN Model}

The equations of hydrodynamics in spherical coordinates and azimuthal symmetry are evolved with a directionally unsplit finite-volume scheme. The scheme is of Godunov type using a second-order-in-space well-balanced reconstruction (Käppeli \& Mishra 2014, 2016) with characteristic limiting, an HLLC approximate Riemann solver (Toro 1997), and a second-order-in-time strong stability-preserving Runge-Kutta (SSP-RK2) (Gottlieb et al. 2001) time integration. Spurious solutions near strong (grid-aligned) shocks are avoided by the use of the H-correction method by Sanders et al. (1998). 

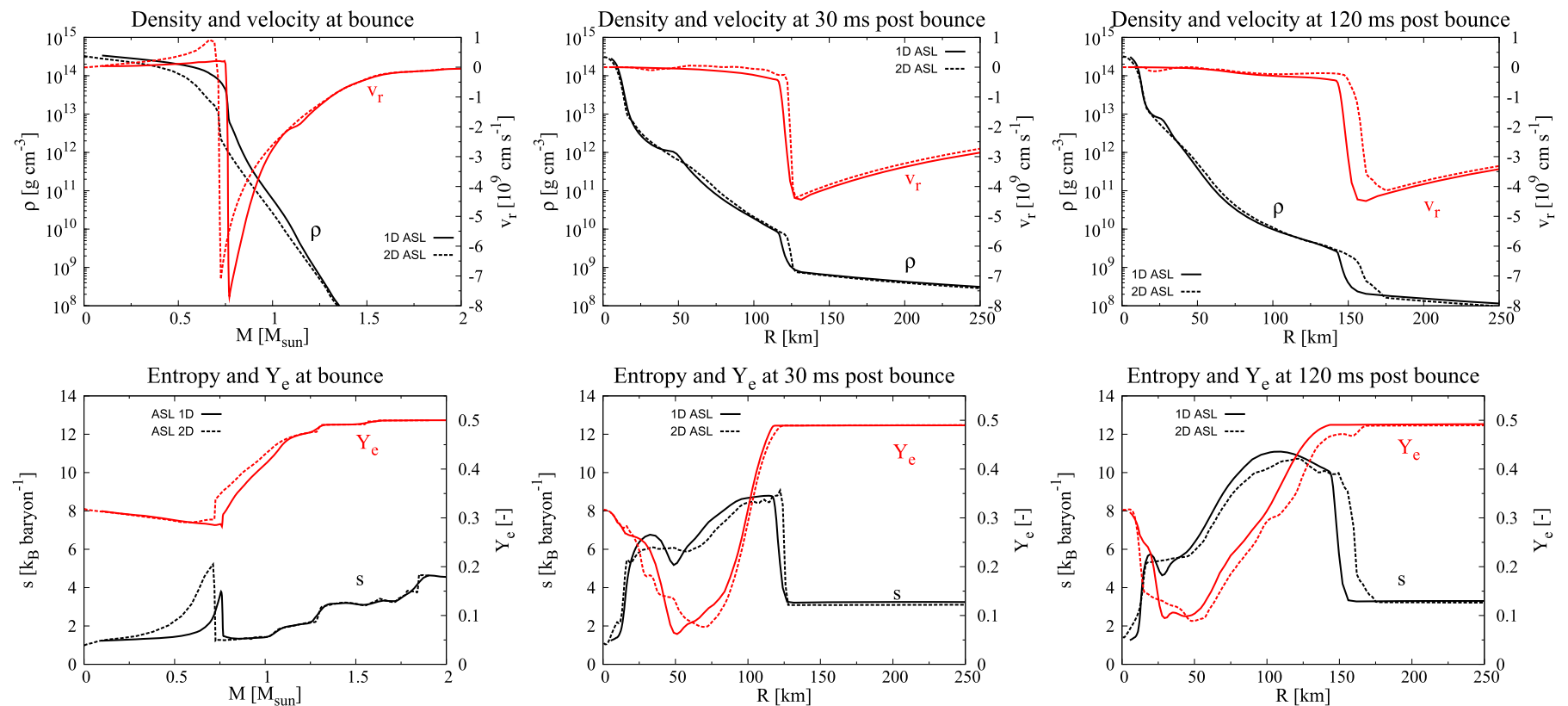

Figure 7. Profiles of the density and radial velocity (top row) and of the entropy and electron fraction (bottom row) for the 1D ASL reference (solid lines) and the spherically averaged 2D ASL model (dashed lines). The left, middle, and right panels refer to 0, 30, and $120 \mathrm{~ms}$ after bounce, respectively.
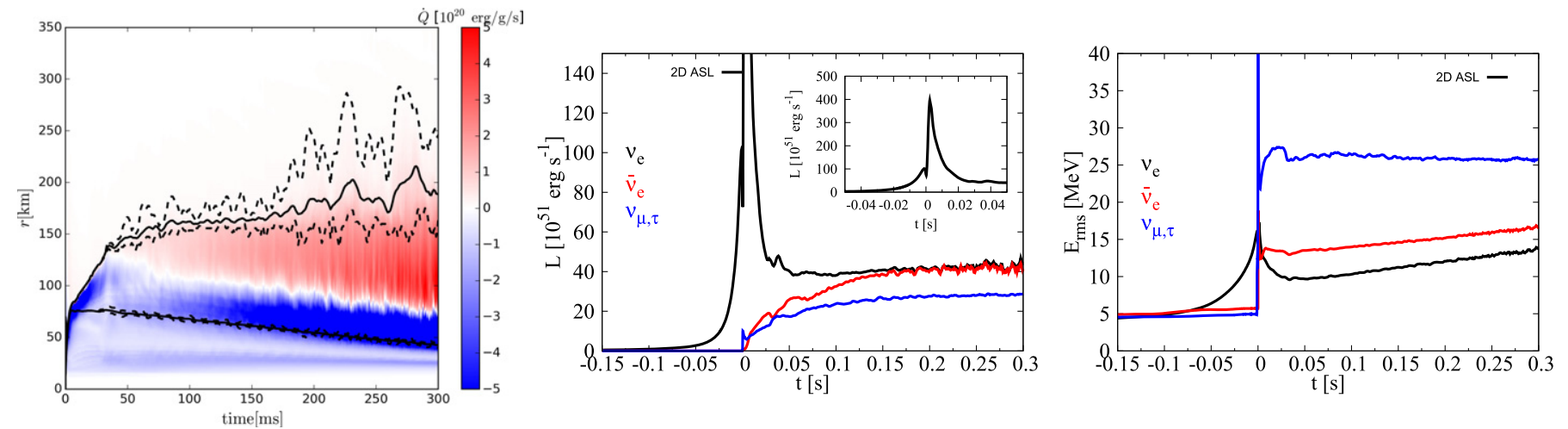

Figure 8. Left: evolution of the PNS and shock radii for the $2 \mathrm{D}$ CCSN model of a $15 M_{\odot}$ progenitor. The solid lines represent the average radius, while the dashed lines represent the minimal and maximal radii, respectively. Furthermore, color-coded is the net energy deposition by the electron flavor neutrinos. Middle: temporal evolution of the neutrino luminosities for the 2D CCSN model. The black, red, and blue lines represent electron neutrinos, electron antineutrinos, and heavy neutrinos, respectively. Right: same as in the middle panel, but for the neutrino rms energies.

Newtonian self-gravity is approximated by a spatially second-order-accurate discretization of the monopole term (i.e., by spherically averaging the mass density and integrating the resulting one-dimensional profile) and a spatially secondorder five-point discretization of the Poisson equation for the deviation from the monopole term.

The ASL scheme is coupled to the hydrodynamics by a "rayby-ray" approach: apart from the trapped neutrino components $\left(Y_{\nu}, Z_{\nu}\right)$, which we evolve according to the corresponding multidimensional advection equations (see Equations (7) and (8)), the ASL scheme is applied as described in Section 2 along each radial "ray." However, in the present implementation we have neglected the neutrino stress in the momentum equation.

The computational domain includes the innermost $5000 \mathrm{~km}$ and the full $[0, \pi]$ polar realm. The radial direction is discretized by $N_{r}=512$ logarithmically spaced cells: $\Delta r_{i}=\Delta r_{1} a^{i}, \quad i=1, \quad \ldots, \quad N_{r}, a-1=5.659 \times 10^{-3}, \quad$ and $\Delta r_{1}=1 \mathrm{~km}$. The polar direction is uniformly discretized by $N_{\theta}=256$ cells. The progenitor is then mapped (without adding any rotation and perturbations) onto the computational domain and evolved numerically through collapse, bounce, and until $300 \mathrm{~ms}$ post-bounce.

The collapse proceeds without any noticeable deviations from spherical symmetry until it is halted by parts of the inner core bouncing at $t_{\text {bounce }}=222 \mathrm{~ms}$ after the start of the simulation owing to the stiffening of the EOS. At that time the central density reaches $\rho_{\text {bounce }}=3.29 \times 10^{14} \mathrm{~g} \mathrm{~cm}^{-3}$. The enclosed mass at the shock formation radius is $M_{\text {enc, }}$ bounce $=0.66 M_{\odot}$, which is slightly lower than in the onedimensional reference simulation. In Figure 7, we present a detailed comparison of the profiles from the axisymmetric simulation with the one-dimensional reference. At bounce (left panels), we observe that all the quantities are in good agreement. The 2D simulation has a slightly more compact PNS, which we attribute to the fact that we have neglected the neutrino stress. At $30 \mathrm{~ms}$ after bounce (middle panels) the agreement is still very good up to the negative entropy gradient, which was washed out by prompt convection. At later times, the two-dimensional simulation deviates from the one-dimensional reference owing to multidimensional effects, e.g., 

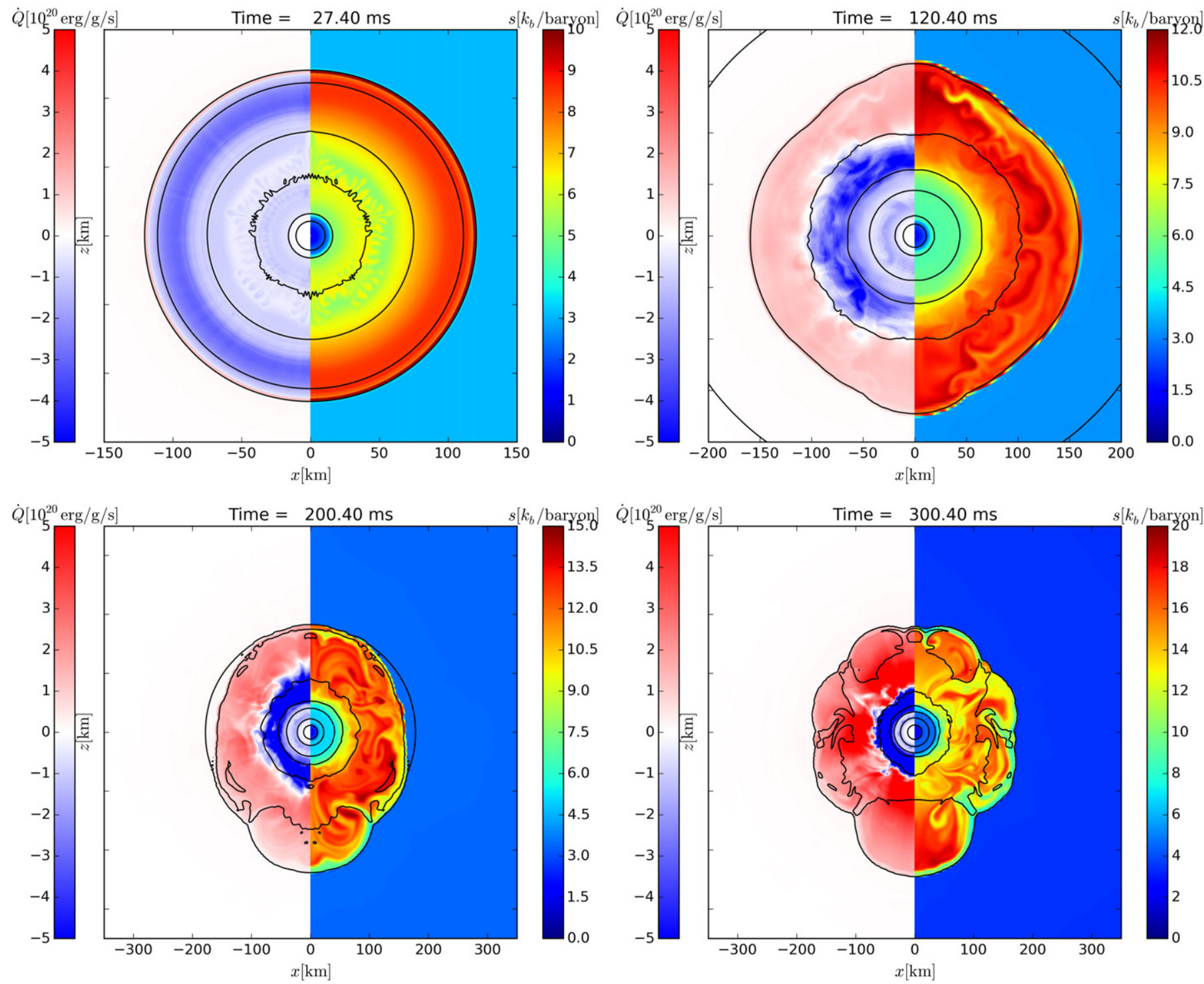

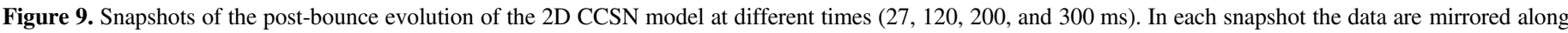
the symmetry axis, displaying on the left the net neutrino heating rate and on the right the specific entropy.

convection and shock instabilities. This is illustrated in the right panels of Figure 7 , where we compare the profiles at $120 \mathrm{~ms}$ after bounce.

In the left panel of Figure 8, we show the minimum, average, and maximum shock and PNS radii. In the same panel, the spherically averaged net heating rate by electron flavor neutrinos is also shown. The shock and PNS radii evolution can be separated into three distinct phases. The first lasts from bounce up to $\approx 30 \mathrm{~ms}$ after bounce. This phase features the initial very strong acceleration of the shock wave. When the shock passes the neutrino spheres, the neutronization burst induces strong cooling as indicated by the negative heating rate. During this phase, the evolution is almost perfectly spherically symmetric. The second phase starts at $\approx 30 \mathrm{~ms}$ after bounce and lasts up to $\approx 170 \mathrm{~ms}$. During this phase, the unstable entropy profile left behind by the shock wave triggers strong convective motions, i.e., the so-called prompt convection. This induces anisotropic shock movements, which are visible in the minimum and maximum shock radii. The effect is also visible in the minimum and maximum PNS radii, but to a much lesser extent. However, the anisotropic shock movements remain mild, i.e., the difference between minimum and maximum shock radius does not exceed $\approx 40 \mathrm{~km}$. In the top left panel of Figure 9, we show a snapshot at $27 \mathrm{~ms}$ postbounce when prompt convection just sets in. During this second phase, one observes the appearance and progressive growth of regions below the shock with net neutrino heating. In the top right panel we show a snapshot at $120 \mathrm{~ms}$ post-bounce. It can be seen that there is an extended heating region below the shock. At this time, convective motions due to neutrino energy deposition start to set in. The third and last phase starts at $\approx 170 \mathrm{~ms}$ and lasts until we stopped the simulation. During this phase, the energy deposited by the neutrinos triggers strong convective motions. Plumes rise by buoyancy against the continuous accretion flow. This in turn triggers increasingly strong shock movements, and it is illustrated in the two bottom panels of Figure 9.

In the middle and right panels of Figure 8, we show the neutrino luminosities and rms energies from the axisymmetric simulation, respectively. Both quantities agree well with the 
one-dimensional reference. Moreover, the multidimensional one shows some oscillations in all the quantities after $\approx 50 \mathrm{~ms}$ post-bounce. These oscillations become stronger after $\approx 150 \mathrm{~ms}$, especially in the luminosities. We attribute these differences again to multidimensional effects.

\section{2. $3 D$ SPH-CCSN Models}

The calculation is performed using our SPH code, SPHYNX, which solves the Euler equations derived from a variational principle (see, e.g., Rosswog 2009, and references therein). SPHYNX uses high-order interpolating kernels, namely, the sinc kernels with $n=5$ (Cabezón et al. 2008; García-Senz et al. 2014), and an improved gradient evaluation based on the integral approach $\mathrm{IAD}_{0}$ (Cabezón et al. 2012; García-Senz et al. 2012; Rosswog 2015a). 3D gravity is calculated with a hierarchical tree structure created using the Barnes-Hut algorithm (Hernquist \& Katz 1989), and the neutrino treatment is handled with a $3 \mathrm{D}$ version of the ASL treatment presented in Section 2.

\subsubsection{SPH + ASL Coupling}

To evolve the system with SPHYNX, we solve the hydrodynamical equations in Lagrangian form, including a gravitational and a neutrino source term. In the momentum equation, we add the total neutrino pressure, Equation (25), to the plasma pressure. Therefore, the stress provided by the trapped neutrinos is directly taken into account. The evolution of each $Z_{\nu}$ is provided by an energy equation (similar to the equation for the plasma internal energy $e$ ), which consistently uses the neutrino pressure $P_{\nu}$.

From the position of the SPH particles and the EOS, we compute the local density, gradient of pressure, gravitational potential, and internal energy. Moreover, each particle carries information regarding the electron fraction, $Y_{e}$, and the neutrino trapped components, $\left(Y_{\nu}, Z_{\nu}\right)$. Similarly to the method presented in Section 2, the ASL scheme ultimately provides the rates of change for these quantities $\left(\dot{Y}_{e}, \dot{Y}_{\nu}, \dot{Z}_{\nu}\right)$ and for the internal energy $\left(\dot{e}_{\nu}\right)$.

The abundances of electrons and neutrinos are evolved explicitly, while the implementation of the energy equation for $Z_{\nu}$ is described in Appendix $\mathrm{C}$.

Most of the quantities that are needed to compute the previous terms are local, so the implementation of the ASL scheme in the SPH structure is straightforward and directly done in 3D with very few modifications of the hydrodynamical part of the code. The only nonlocal quantities are the spectral optical depths, $\tau_{\nu \text {,tot }}(\boldsymbol{x}, E)$ and $\tau_{\nu \text {, en }}(\boldsymbol{x}, E)$, and the spectral neutrino densities, $n_{\nu}(E, \boldsymbol{x})$, used in the calculation of the nonlocal absorption rates, $h_{\nu}(E, \boldsymbol{x})$ (Equation (35)).

To compute the optical depths, we use the expected quasispherical symmetry of a collapsing stellar core by defining a one-dimensional radial grid. On this grid we calculate the spherical averages of the neutrino spectral mean free paths (which are computed locally in $3 \mathrm{D}$, at each SPH particle position). Then, we integrate $1 / \lambda(R, E)$ radially, from the external edge up to each radial position, to obtain the radial optical depth. Finally, the spherically symmetric optical depth is mapped back on the 3D SPH particle distribution, interpolating with respect to the distance from the center of mass. Using the $3 \mathrm{D}$ density as the interpolation variable led to no significant differences. Regarding the neutrino densities, we also consider them to be spherically symmetric, and to evaluate them, we calculate the spectral number luminosity via performing a radial integration over all the particles sorted from lower to higher radius.

\subsubsection{Setup and Results}

We set up a CCSN SPH simulation with 200,000 particles, using Newtonian gravity. We map the spherically symmetric progenitor model into a 3D quasi-random Sobol distribution of equal-mass particles. We simulate $\sim 1.8 M_{\odot}$ of mass and up to $3800 \mathrm{~km}$ in radius. Next, we perform an angular relaxation of the system by allowing the particles to move, but with fixed radius. In this way we erase artificial gradients of pressure formed by random clumps of particles, and we obtain clean radial profiles that adjust to the initial 1D model.

The dynamics of the collapse and of the early post-bounce phase are in very good agreement with the previous results. The electron fraction in the center of the PNS decreases until it reaches $Y_{e} \sim 0.31$. When the central density becomes $\rho_{\text {bounce }}=3.3 \cdot 10^{14} \mathrm{~g} \mathrm{~cm}^{-3}$, the core bounces and a shock wave forms at the surface of the newly born PNS. More specifically, the shock is formed at $\approx 14 \mathrm{~km}$, which corresponds to an enclosed mass of $M_{\text {enc,bounce }}=0.76 M_{\odot}$ of unshocked material.

In Figure 10, we present radial profiles of radial velocity (left column), electron fraction (central column), and entropy (right column), at four different times from core bounce up to $100 \mathrm{~ms}$. Each point in the panels represents one of the SPH particles. From the low dispersion of the profiles at bounce, we can see that the collapse preserves spherical symmetry, as expected. We notice the position of the shock wave from the entropy spike and from the corresponding starting deleptonization in the $Y_{e}$ profile. As the shock proceeds, multidimensional effects and inhomogeneities appear and grow inside the shocked material. This effect can be seen from the spread of particles in the profiles of all represented quantities in the second row. After $50 \mathrm{~ms}$, the initial prompt convection has been quenched and the evolution proceeds more steadily. Later on, convection slowly settles again in the shocked material, but with a longer timescale, which can be seen in the profiles of radial velocity and $Y_{e}$ as an increased scatter of the particles (last row). The shape and the evolution of multidimensional instabilities can be better seen in Figure 11. In this series of snapshots, we project onto the $x y$ plane all the particles included in a thin layer of $20 \mathrm{~km}$ around the equatorial plane $(Z=0)$, for the same time steps used in Figure 10. The size of the box is $200 \mathrm{~km}$ wide. Material processed by the shock is slowed down, deleptonized, and accreted onto the hot PNS. At $17 \mathrm{~ms}$ we show the development of a transitory violent convection that comes right after the shock launch, which in this snapshot has reached the outer edge of the plot, at $R \simeq 100 \mathrm{~km}$. At $50 \mathrm{~ms}$ the convection modes have been quenched and the PNS accretes with a steady flow. This leads to the more evolved image at $100 \mathrm{~ms}$, where accretion occurs smoothly on a slowly compactifying PNS. As time proceeds, the accretion and compression of matter increase the temperature above the surface where the shock was launched. The low numerical diffusion of SPH helps keep the heat located in this region. The strong deleptonization via neutrino emission can be seen at work in all snapshots, even at $17 \mathrm{~ms}$, where evident 3D features occur, showing that the ASL scheme is working correctly in multidimensional simulations. 

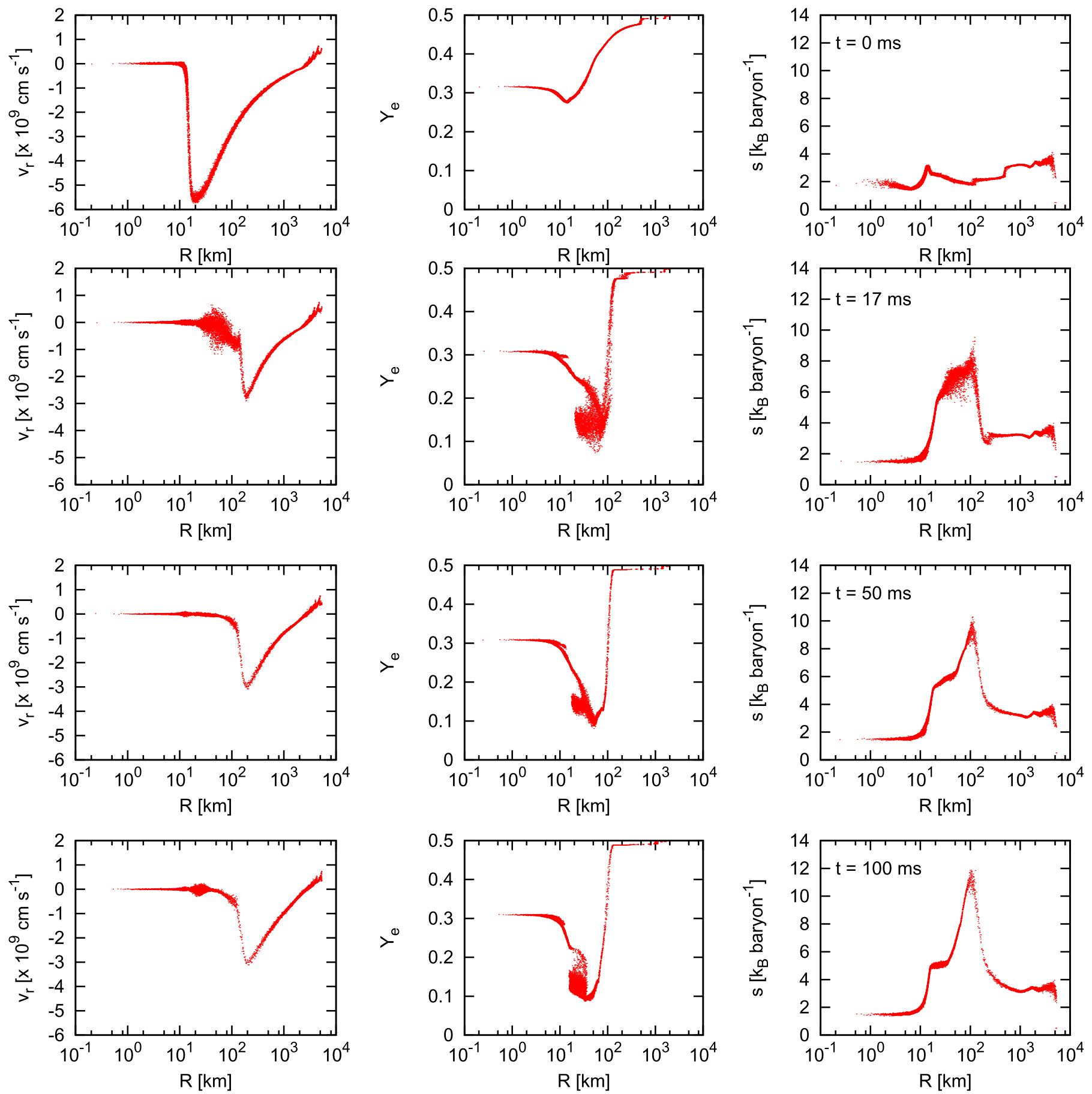

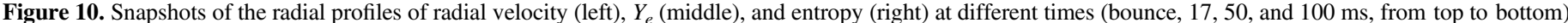
from our SPH model of a $15 M_{\odot}$ CCSN. Each point represents one SPH particle, and only 1 out of every 10 is plotted.

In the left panel of Figure 12, we show the evolution of the PNS radius (black line) and of the shock radius (red line) as a function of time after bounce. The PNS shows a stable evolution with a slowly decreasing radius, corresponding to a more compactified configuration due to accretion and cooling. The shock position is determined within the local resolution as the radius at which the artificial viscosity (AV) peaks. The AV is specifically designed to dissipate energy only when shocks are present. We saw in all of our simulations that, once the shock is formed and launched, the AV is approximately two orders of magnitude higher at the shock position than in the surrounding matter. The overall evolution of the shock is similar to the spherical results, with a fast expansion reaching $100 \mathrm{~km}$ within the first $5 \mathrm{~ms}$, and after some oscillations, triggered by 3D convection, it settles around $150 \mathrm{~km}$. In the same figure, we also present the time evolution of the local cooling and heating rates color-coded. The thin white region between the cooling (blue) and the heating (red) regions is the location of the gain radius, which settles around $100 \mathrm{~km}$ at $50 \mathrm{~ms}$ after bounce and slowly recedes, while the neutrino heating sets in behind the shock. 

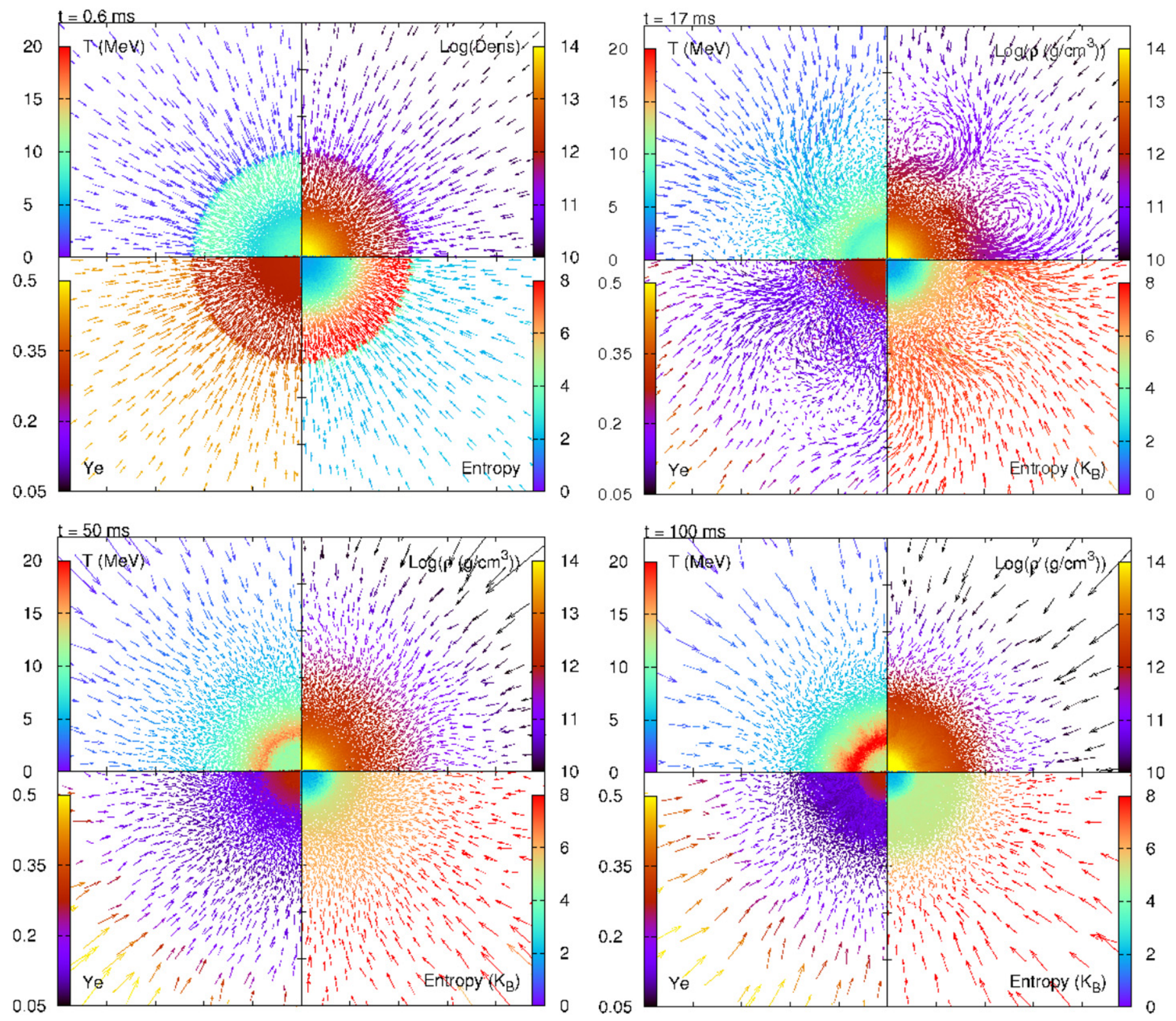

Figure 11. Snapshots of the post-bounce evolution at different times $(0.6,17,50$, and $100 \mathrm{~ms})$ from our SPH model of a $15 M_{\odot}$ CCSN. We show here only a thin slice of the 3D domain on the $x y$ plane, and each box is $200 \mathrm{~km}$ wide, i.e., $-100 \mathrm{~km}<x, y<100 \mathrm{~km}$. Each arrow represents one SPH particle and shows its projected velocity. Temperature, density, $Y_{e}$, and entropy are color-coded in each snapshot.
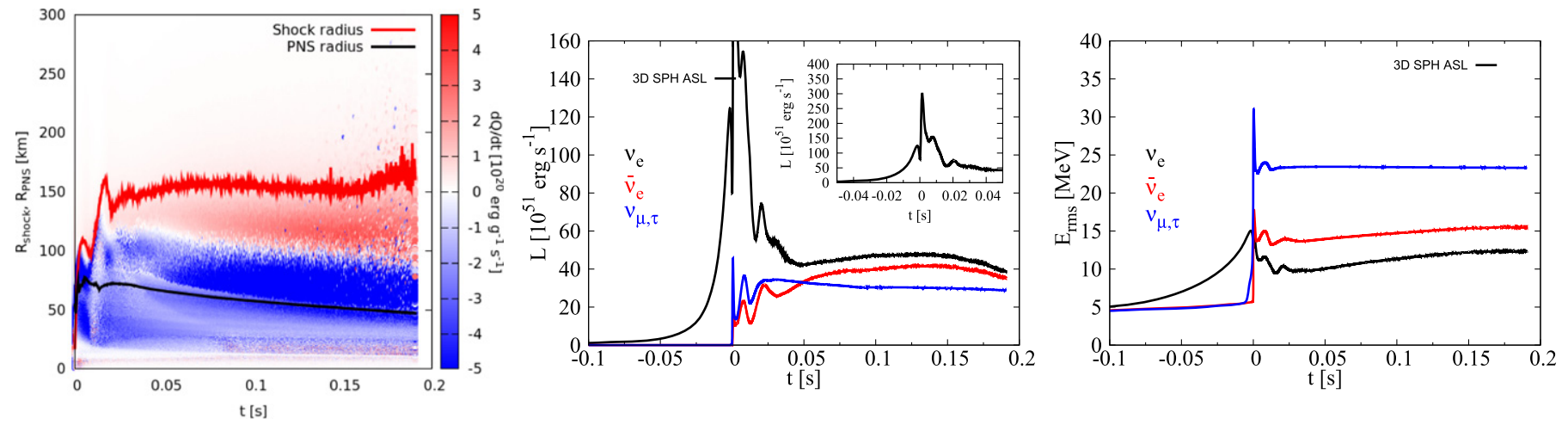

Figure 12. Same as in Figure 8, but for our 3D SPH simulation of a $15 M_{\odot}$ CCSN. 
In the middle and right panels of Figure 12, we show the temporal evolution of the neutrino luminosities and rms energies, respectively, calculated during our SPH simulation with the ASL scheme. The obtained results agree well with the 1D simulation performed with the ASL scheme. In particular, in the luminosity evolutions we distinguish all relevant features expected in the collapse, burst, and accretion phases. Also, the rms energies have an evolution that corresponds very well with the results obtained with the reference 1D model, and their hierarchy is preserved through the simulation. Another feature that looks interesting is the oscillation in the luminosities within the first milliseconds after neutrino burst: they appear in all three curves, and they are related to violent convection inside the PNS during the very early post-bounce phase. Prompt, violent convection inside the PNS was also observed, for example, by Herant et al. (1994) in 2D SPH simulations, and more recently by Dolence et al. (2015) and Pan et al. (2015). This feature deserves a deeper investigation (see, e.g., the analysis performed by Bruenn et al. 2004; Buras et al. 2006a).

\section{CONCLUSIONS}

We have presented the ASL scheme. This provides an approximate treatment for the neutrino transport problem in astrophysical contexts, like the core collapse of a massive star or the merger of compact objects. The goal of the scheme is to provide an efficient and physically motivated treatment that contains all the major aspects of neutrino emission and absorption, with a level of accuracy lower than other more sophisticated (multidimensional) neutrino transports (like, for example, M1 schemes, MGFLD schemes, or IDSA), but higher than the classical gray leakage schemes. It allows the application to different astrophysical contexts, codes, and geometrical dimensions, with a reduced computational cost. Owing to its effective nature, details of the neutrino transport cannot always be reproduced. Nevertheless, it is optimal (1) to study problems where a spectral neutrino treatment is required, but the details of the neutrino behavior are of secondary importance; (2) to perform extensive parametric or highresolution studies, which are still computationally too costly in multidimensional simulations with detailed neutrino transports; (3) to accomplish preparatory and exploratory tests; (4) during the developing and testing of a hydrodynamic code, when the usage of an easily verifiable but still reliable neutrino treatment could be useful; and (5) to study complex and very dynamical systems in which, due to the lack of symmetries, other more sophisticated neutrino treatments are still not available.

Owing to its approximate nature, it is not well suited to investigate aspects where the details of the neutrino transport are crucial (e.g., Lentz et al. 2012a, 2012b; Müller et al. 2012b; Melson et al. 2015). Moreover, since it avoids the solution of the transport problem in the diffusive regime by estimates of the diffusion timescales, it is not designed to study the detailed cooling of compact objects, especially over long timescales (Hüdepohl et al. 2010; Fischer et al. 2012; Roberts et al. 2012; Suwa 2014).

We have developed and tested the scheme against reference models provided by numerical solutions of the Boltzmann equation. We have explored three progenitors with 12,15 , and $40 M_{\odot}$ ZAMS masses. The $15 M_{\odot}$ case has been more extensively studied to calibrate the free parameters of the scheme. We have also investigated the impact of the variation of their values on the obtained results. Usually, the changes we have tested produced differences qualitatively in agreement with what we expected. Large quantitative discrepancies are observed when the parameter values differ significantly from the calibrated ones. The 12 and $40 M_{\odot}$ cases have been used to show the robustness of the calibrated parameters with respect to the progenitor model.

Overall, the radial profiles of several hydrodynamical and thermodynamical quantities obtained by the ASL scheme show a good agreement with the reference solutions during the whole simulated period (from the collapse to the neutrino heating phase). Small differences are present during the collapse phase and in the prompt shock expansion, while in the neutrino heating phase, where a detailed treatment would be required to model with accuracy both the neutrino emission and absorption, differences tend to grow. Usually, the shock position is well reproduced, with typical differences not larger than $10-15 \mathrm{~km}$ even at later times (larger discrepancies have been observed only for the $12 M_{\odot}$ case). The profiles of electron fraction and entropy are the ones that present the most notable differences, even though some differences can be interpreted as radial or temporal shifts. We have also compared the temporal evolution of the neutrino luminosities and mean energies, for all neutrino flavors. Again, the most relevant features are present also in the approximate results, especially for $\nu_{e}$. Quantitative differences are nevertheless visible, especially in the rapid growth of $\bar{\nu}_{e}$ and $\nu_{\mu, \tau}$ in the early post-bounce phase. The behaviors and the values we have obtained for the rms energies of $\nu_{e}$ and $\bar{\nu}_{e}$ are consistent with the reference solution. This is true especially in the neutrino heating phase, where correctly describing the mean neutrino energies is crucial to model the neutrino absorption in the optically thin region.

We have also shown that the scheme can be applied, without conceptual changes, to different types of codes and to different spatial dimensions. In this respect, we have implemented and tested it in multidimensional core-collapse models using an axisymmetric Eulerian grid code and a 3D Lagrangian SPH code. These models agree with our spherically symmetric solution during the collapse and in the early post-bounce phase. Multidimensional features appear in the post-bounce phase, and they agree (at least, at a qualitative level) with published results obtained in multidimensional models employing more detailed neutrino transport. In fact, the ASL treatment has already been applied to multidimensional CCSN models (Winteler et al. 2012; Perego et al. 2015) and to the study of the aftermath of neutron star mergers (Perego et al. 2014b).

The scheme presents a modular structure that allows the inclusion of new neutrino reactions and opacities, as well as the possibility to include more sophisticated treatments (for example, for the reconstruction of the trapped distribution functions or for the neutrino thermalization process), without changing its basic features. The inclusion of additional neutrino reactions, like NES, or of some relevant relativistic and Doppler effects (e.g., Lentz et al. 2012b, and references therein) in the ASL scheme, will be carried out in the nearby future.

The authors thank A. Arcones, E. Gafton, M. Liebendörfer, S. Rosswog, and F.-K. Thielemann for useful discussions and comments about this work. A.P. is supported by the HelmholtzUniversity Investigator grant No. VH-NG-825. He also thanks the University of Basel and ETH Zürich for their hospitality. R. C. acknowledges the support from the HP2C Supernova 
project, the European Research Council (FP7) under the ERC Advanced Grant Agreement N 321263-FISH, and the DIAPHANE project within the Platform for Advanced Scientific Computing (PASC). R.C. and R.K. thank TU Darmstadt for its hospitality. A.P., R.C., and R.K. acknowledge the use of computational resources provided by the Swiss SuperComputing Center (CSCS), under the allocation grant s414. The authors thank also $M$. Liebendörfer for the access to BOLTZTRAN runs and acknowledge the support of the sciCORE (http://scicore.unibas.ch/) scientific computing core facility at the University of Basel, where some of the calculations were performed. R.K. acknowledges the computing resources provided by the Brutus and Euler clusters at ETHZ.

\section{APPENDIX A \\ IMPLEMENTATION OF THE PAIR PROCESSES IN THE ASL SCHEME}

In this appendix, we present our implementation of the neutrino pair processes, Equations (45) and (46), in the context of the ASL scheme. Our goal is the computation of the associated emissivities, $\left(j_{\nu}\right)_{\text {pair }}$, and absorptivities, $\left(\chi_{\nu}\right)_{\text {pair }}$. These quantities are necessary to compute the local mean free paths, Equations (1) and (2), the production and diffusion timescales, Equations (21) and (22), and the production rates, Equation (30). We start from the expression of the collision integral for pair processes in the Boltzmann equation for the neutrino species $\nu$ (see, e.g., Bruenn 1985; Hannestad \& Raffelt 1998):

$$
\begin{aligned}
\left.\dot{f}_{\nu}\left(\boldsymbol{k}_{\nu}\right)\right|_{\text {coll,pair }}= & \left(1-f_{\nu}\left(\boldsymbol{k}_{\nu}\right)\right) \frac{1}{c(2 \pi \hbar c)^{3}} \int d^{3} \boldsymbol{k}_{\bar{\nu}} \\
& \times\left(1-f_{\bar{\nu}}\left(\boldsymbol{k}_{\bar{\nu}}\right)\right) S_{\text {pair }}^{\text {pr }}\left(\boldsymbol{k}_{\nu}, \boldsymbol{k}_{\bar{\nu}}\right) \\
& -f_{\nu}\left(\boldsymbol{k}_{\nu}\right) \frac{1}{c(2 \pi \hbar c)^{3}} \int d^{3} \boldsymbol{k}_{\bar{\nu}} f_{\bar{\nu}} \\
& \times\left(\boldsymbol{k}_{\bar{\nu}}\right) S_{\text {pair }}^{\mathrm{ab}}\left(\boldsymbol{k}_{\nu}, \boldsymbol{k}_{\bar{\nu}}\right)
\end{aligned}
$$

where $\bar{\nu}$ denotes the antiparticle of $\nu, \boldsymbol{k}_{\nu}$ and $\boldsymbol{k}_{\bar{\nu}}$ the neutrino momenta, $f_{\nu}$ and $f_{\bar{\nu}}$ the neutrino distribution functions, and $S_{\text {pair }}^{\mathrm{pr}}$ and $S_{\text {pair }}^{\text {ab }}$ the kernel of the pair reactions.

To compute the local emissivities, we consider the first term in the integral of Equation (49) and perform the integral over the $\bar{\nu}$ phase space, neglecting Pauli blocking factors for $\bar{\nu}$ in the final state, since the production rate is mainly relevant in the optically thin region:

$$
j_{\text {em,pair }}\left(E_{\nu}\right)=\frac{1}{c(2 \pi \hbar c)^{3}} \int d^{3} \boldsymbol{k}_{\bar{\nu}} S_{\text {pair }}^{\mathrm{pr}}\left(\boldsymbol{k}_{\nu}, \boldsymbol{k}_{\bar{\nu}}\right) .
$$

For the absorptivities, we consider the right-hand term of the integral in Equation (49), and we integrate it over the phase space of $\bar{\nu}$,

$$
\chi_{\text {ab,pair }}\left(E_{\nu}\right)=\frac{1}{c(2 \pi \hbar c)^{3}} \int d^{3} \boldsymbol{k}_{\bar{\nu}} f_{\bar{\nu}}\left(\boldsymbol{k}_{\bar{\nu}}\right) S_{\text {pair }}^{\mathrm{ab}}\left(\boldsymbol{k}_{\nu}, \boldsymbol{k}_{\bar{\nu}}\right),
$$

assuming that $f_{\bar{\nu}}$ is described by Fermi-Dirac distribution functions in weak and thermal equilibrium. Within this assumption, we recover the correct limit in the diffusive regime, where the calculations of the mean free path and of the optical depth are more relevant. In the optically thin limit, where the actual distribution functions are expected to differ significantly from Fermi-Dirac distributions, our approach is expected to overestimate the absorptivity due to pair processes. This would lead to a smaller $\lambda_{\text {pair }}$. However, we have tested that, for the corresponding relevant thermodynamical conditions, $\lambda_{\text {pair }}$ is still significantly larger than the mean free path owing to absorption or scattering on nucleons. Moreover, $\lambda_{\text {pair }}$ is always much larger than the linear dimension of the system. Thus, our overestimated absorptivities in optically thin conditions do not affect critically the location of the neutrino surfaces.

The reaction kernels, $S_{\text {pair }}^{\text {ab }}$ and $S_{\text {pair }}^{\text {pr }}$, are calculated following Hannestad \& Raffelt (1998) for the bremsstrahlung process and Bruenn (1985) and Mezzacappa \& Messer (1999) for the pair annihilation process. The calculation of the integrals (50) and (51) during runtime would be by far the most expensive part of the rate computation. Thus, we decide to tabulate these emission and absorption rates and to interpolate them during the program execution. We compute them as a function of the matter density, electron fraction, and temperature. For our tables we consider a 3D grid, where we uniformly sample (i) the logarithm of the matter density, with 104 points between $10^{5.1}$ and $10^{15.4} \mathrm{~g} \mathrm{~cm}^{-3}$; (ii) the electron fraction, with 72 points between 0.0 and 0.56 ; (iii) the logarithm of the matter temperature, with 31 points between 0.1 and $100 \mathrm{MeV}$. We perform the integral over the neutrino energy by splitting the $[0,+\infty)$ integration interval into two segments, $\left[0, E_{\text {ref }}\right)$ and $\left[E_{\text {ref }},+\infty\right)$, with $E_{\text {ref }}=3 T / 2$. For the second, improper integral, we perform the change of variable $E \rightarrow 1 / E$. Then, we integrate each of the two integrals using the GaussLegendre quadrature with 16 points. The interpolation at runtime is accomplished by a trilinear interpolation method.

\section{APPENDIX B \\ PERFORMANCE COMPARISON}

To analyze the performance differences between Agile-ASL and Agile-BOLTZTRAN, we consider the reference runs presented in Section 3 for the $15 M_{\odot}$ progenitor (Woosley et al. 2002), from the onset of the collapse up to $300 \mathrm{~ms}$ after core bounce. Agile solves the hydrodynamics equation in an implicit way. In principle, this allows the usage of Courant numbers larger than 1 in the determination of the physical time step. This is the case for Agile-BOLTZTRAN since BOLTZTRAN is also implemented in an implicit way. On the other hand, the ASL scheme mimics a diffusion process and is implemented in an explicit way. Therefore, when we combine Agile with the ASL scheme, we limit the time step by requiring a Courant number lower than 0.75 to ensure a tight enough coupling. Moreover, if necessary, we further adjust the time step such that the maximum relative variation in $Y_{e}$ induced by the neutrino source terms is always below $5 \times 10^{-3}$ per step. An increase of the tolerance up to $10^{-2}$ per step produces no noticeable differences in the results.

We additionally extend the comparison to IDSA, which is a very efficient, approximated $\nu_{e} / \bar{\nu}_{e}$ transport scheme. For this comparison we use the publicly available version of the code for spherically symmetric CCSN models, ${ }^{7}$ which is also coupled with Agile and includes the cooling effect of $\nu_{\mu, \tau}$ and $\bar{\nu}_{\mu, \tau}$ via a gray leakage scheme. We run it for the same progenitor and code setup. To compare directly with the ASL

\footnotetext{
https://physik.unibas.ch/ liebend/download/index.html
} 

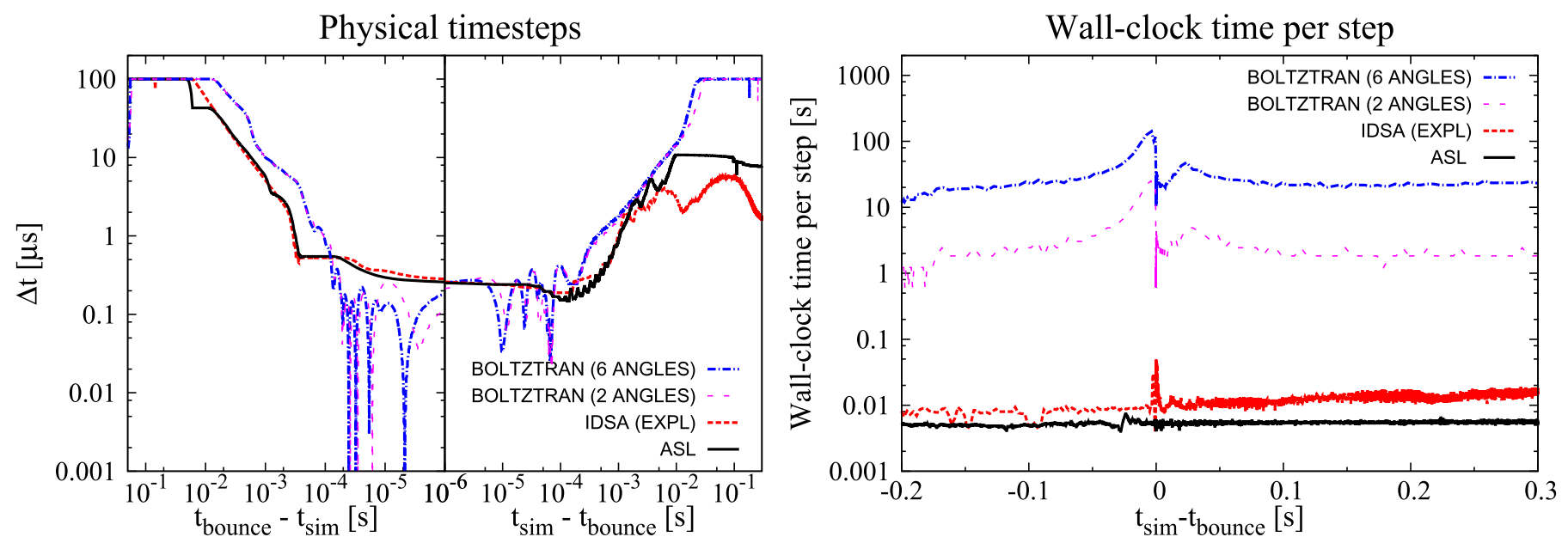

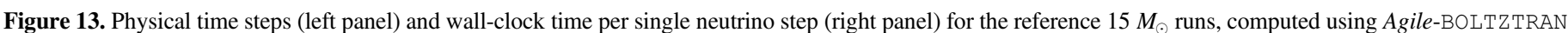

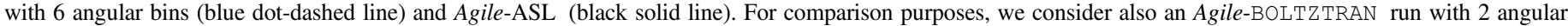

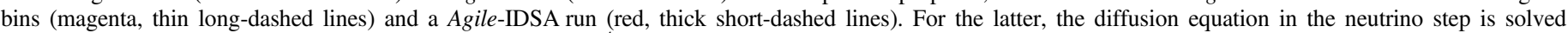

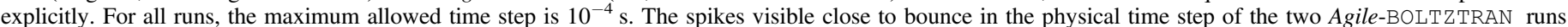
represent a few oscillations between $10^{-7}$ and $10^{-13} \mathrm{~s}$, lasting only a few thousands of iterations.

scheme, the solution of the diffusion equation inside IDSA is performed in an explicit way.

We perform all runs on a single processor. In Figure 13, we present the physical time step used by the three different codes (left panel) and the wall-clock time per step spent by each code to perform one neutrino step (right panel). Since the performances of Agile-BOLTZTRAN depend significantly on the accuracy of the solution, we include in the comparison also a run where we have decreased the number of bins in the neutrino propagation angle from 6 to 2. From the time step profiles, we notice that the implicit character of AgileBOLTZTRAN allows larger time steps (up to a factor of 10-50) both in the collapse and in the post-bounce phase, compared with Agile-ASL and the explicit version of AgileIDSA. However, in the right panel we clearly see that the solution of the Bolztmann equation requires much larger wallclock times per step, of the order of $10^{3}-10^{4}$ times larger than those of Agile-ASL for 6 angular bins, and still $10^{2}-10^{3}$ times larger for 2 angular bins. Moreover, despite the fact that IDSA models two independent neutrino species while the ASL scheme three species, we observe that the former requires roughly double wall-clock time to perform one neutrino step compared with the latter.

\section{APPENDIX C \\ CALCULATION OF THE ENERGY DENSITY OF NEUTRINOS IN SPHYNX}

In this appendix, we present our implementation of the equation that evolves $Z_{\nu}$ in the SPH implementation of the ASL scheme. In the following, all SPH equations use $\mathrm{IAD}_{0}$ for calculating derivatives (Cabezón et al. 2012; García-Senz et al. 2012; Rosswog 2015a). To make a conversion to the traditional SPH prescription, simply substitute all $\mathcal{A}_{i j}$ by $\nabla_{i} W_{i j}$.

The trapped neutrino energy $Z_{\nu}$ is evolved according to

$$
\frac{d Z_{\nu}}{\mathrm{dt}}=-\frac{P_{\nu}}{\rho} \nabla \cdot \boldsymbol{v}+\dot{Z}_{\nu}
$$

From Equation (52) it is clear that the variation of $Z_{\nu}$ consists of two contributions: The first term is due to the $\operatorname{Pd} V$ work and can be calculated using an SPH equation similar to the SPH (baryonic) energy equation, which takes into account the neutrino pressure (instead of the baryonic pressure) and the density changes of the fluid. The second one is the source term provided by the ASL scheme and takes into account the rate of change of energy of the trapped neutrinos due to production and diffusion. Overall, evolving independent equations for $\dot{Z}_{\nu}$ is equivalent to splitting the equation for the total internal energy $u$ into two components: baryonic and neutrinos. The baryonic part is accounted for with a regular SPH equation for specific internal energy $e$, while the neutrino component is calculated with

$$
\left(\frac{d Z_{\nu}}{d t}\right)_{i}=\frac{P_{\nu, i}}{\Omega_{i} \rho_{i}^{2}} \sum_{j} m_{j}\left(\boldsymbol{v}_{i}-\boldsymbol{v}_{j}\right) \cdot \mathcal{A}_{i j}+\dot{Z}_{\nu, i}
$$

where $i$ is the particle index, $v$ is the velocity vector, and $\Omega_{i}$ is the grad-h term. Then, $Z_{\nu}^{n+1}$ could be calculated using the same integration method used for the specific internal energy.

Although this scheme is quite straightforward, in order to calculate the new $Z_{\nu}$, we evaluate the rate of change $\dot{Z}_{\nu}$, which in fact depends on $Z_{\nu}$ itself via $P_{\nu}$ (Equation (25)). Therefore, we opted for developing a semi-implicit scheme that preserves the consistency between both magnitudes at a very low computational cost. Noting that our objective is

$$
P_{\nu}^{n+1}=\frac{1}{3} \rho Z_{\nu}^{n+1},
$$

we can now substitute Equation (54) into Equation (53) to explicitly show its dependence on $Z_{\nu}^{n+1}$. Taking into account all sources, we can write

$$
Z_{\nu, i}^{n+1}=Z_{\nu, i}^{n}+\dot{Z}_{\nu, i}^{n} \Delta t+\frac{1}{3} \frac{Z_{\nu, i}^{n+1}}{\Omega_{i} \rho_{i}} \sum_{j} m_{j}\left(\boldsymbol{v}_{i}-\boldsymbol{v}_{j}\right) \cdot \mathcal{A}_{i j} \Delta t .
$$

Regrouping and isolating $Z_{\nu, i}^{n+1}$ on the left-hand side, we obtain the final version for the evolution of $Z_{\nu, i}$ for each 
neutrino species:

$$
Z_{\nu, i}^{n+1}=\frac{Z_{\nu, i}^{n}+\dot{Z}_{\nu, i}^{n} \Delta t}{1-\frac{1}{3} \frac{1}{\Omega_{i} \rho_{i}} \sum_{j} m_{j}\left(\boldsymbol{v}_{i}-\boldsymbol{v}_{j}\right) \cdot \mathcal{A}_{i j} \Delta t} .
$$

Equation (56) shows a very stable evolution for $Z_{\nu}$ in all the simulations, and it provides consistent values for the neutrino pressure and the neutrino energy by construction. After its evaluation, we use the new $Z_{\nu}^{n+1}$ to calculate, via Equation (54), the neutrino pressure $P_{\nu}$ that is afterward included in the momentum equation via adding it to the baryonic pressure.

\section{REFERENCES}

Abdikamalov, E., Burrows, A., Ott, C. D., et al. 2012, ApJ, 755, 111 Arnett, W. D. 1977, ApJ, 218, 815

Bludman, S. A., Lichtenstadt, I., \& Hayden, G. 1982, ApJ, 261, 661 Bowers, R. L., \& Wilson, J. R. 1982, ApJS, 50, 115

Brandt, T. D., Burrows, A., Ott, C. D., \& Livne, E. 2011, ApJ, 728, 8 Bruenn, S. W. 1985, ApJS, 58, 771

Bruenn, S. W., Lentz, E. J., Hix, W. R., et al. 2016, ApJ, 818, 123 Bruenn, S. W., Mezzacappa, A., Hix, W. R., et al. 2013, ApJL, 767, L6 Bruenn, S. W., Raley, E. A., \& Mezzacappa, A. 2004, arXiv:astro-ph/0404099 Buras, R., Janka, H.-T., Rampp, M., \& Kifonidis, K. 2006a, A\&A, 457, 281 Buras, R., Rampp, M., Janka, H.-T., \& Kifonidis, K. 2006b, A\&A, 447, 1049 Burrows, A. 2013, RvMP, 85, 245

Burrows, A., Dessart, L., Livne, E., Ott, C. D., \& Murphy, J. 2007, ApJ, 664, 416

Burrows, A., Young, T., Pinto, P., Eastman, R., \& Thompson, T. A. 2000, ApJ, 539, 865

Cabezón, R. M., García-Senz, D., \& Escartín, J. A. 2012, A\&A, 545, A112

Cabezón, R. M., García-Senz, D., \& Relaño, A. 2008, JCoPh, 227, 8523

Cooperstein, J. 1988, PhR, 163, 95

Cooperstein, J., van den Horn, L. J., \& Baron, E. A. 1986, ApJ, 309, 653

Cooperstein, J., van den Horn, L. J., \& Baron, E. A. 1987, ApJ, 315, 729

Couch, S. M., \& Ott, C. D. 2013, ApJL, 778, L7

Deaton, M. B., Duez, M. D., Foucart, F., et al. 2013, ApJ, 776, 47

Dessart, L., Burrows, A., Livne, E., \& Ott, C. D. 2006, ApJ, 645, 534

Dessart, L., Ott, C. D., Burrows, A., Rosswog, S., \& Livne, E. 2009, ApJ, 690,1681

Dolence, J. C., Burrows, A., \& Zhang, W. 2015, ApJ, 800, 10

Faber, J. A., \& Rasio, F. A. 2012, LRR, 15, 8

Fernández, R. 2012, ApJ, 749, 142

Fernández, R., \& Metzger, B. D. 2013, MNRAS, 435, 502

Fischer, T., Martínez-Pinedo, G., Hempel, M., \& Liebendörfer, M. 2012, $\mathrm{PhRvD}, 85,083003$

Fischer, T., Whitehouse, S. C., Mezzacappa, A., Thielemann, F.-K., \& Liebendörfer, M. 2010, A\&A, 517, A80

Foglizzo, T., Kazeroni, R., Guilet, J., et al. 2015, PASA, 32, 9

Foucart, F., Haas, R., Duez, M. D., et al. 2016, PhRvD, 93, 044019

Foucart, F., O'Connor, E., Roberts, L., et al. 2015, PhRvD, 91, 124021

Fryer, C. L. 1999, ApJ, 522, 413

Fryer, C. L., \& Warren, M. S. 2004, ApJ, 601, 391

Galeazzi, F., Kastaun, W., Rezzolla, L., \& Font, J. A. 2013, PhRvD, 88, 064009

García-Senz, D., Cabezón, R. M., \& Escartín, J. A. 2012, A\&A, 538, A9

García-Senz, D., Cabezón, R. M., Escartín, J. A., \& Ebinger, K. 2014, A\&A, 570, A14

Gottlieb, S., Shu, C.-W., \& Tadmor, E. 2001, SIAMR, 43, 89

Handy, T., Plewa, T., \& Odrzywołek, A. 2014, ApJ, 783, 125

Hanke, F., Marek, A., Müller, B., \& Janka, H.-T. 2012, ApJ, 755, 138

Hannestad, S., \& Raffelt, G. 1998, ApJ, 507, 339

Herant, M., Benz, W., Hix, W. R., Fryer, C. L., \& Colgate, S. A. 1994, ApJ, 435,339

Hernquist, L., \& Katz, N. 1989, ApJS, 70, 419

Hüdepohl, L., Müller, B., Janka, H.-T., Marek, A., \& Raffelt, G. G. 2010, PhRvL, 104, 251101

Janka, H.-T. 1992, A\&A, 256, 452

Janka, H.-T. 2001, A\&A, 368, 527

Janka, H.-T. 2012, ARNPS, 62, 407

Janka, H.-T., \& Hillebrandt, W. 1989a, A\&AS, 78, 375

Janka, H.-T., \& Hillebrandt, W. 1989b, A\&A, 224, 49

Just, O., Obergaulinger, M., \& Janka, H.-T. 2015, MNRAS, 453, 3386

Käppeli, R., \& Mishra, S. 2016, A\&A, 587, 94
Käppeli, R., \& Mishra, S. 2014, JCoPh, 259, 199

Käppeli, R., Whitehouse, S. C., Scheidegger, S., Pen, U.-L., \& Liebendörfer, M. 2011, ApJS, 195, 20

Kiuchi, K., Sekiguchi, Y., Kyutoku, K., \& Shibata, M. 2012, CQGra, 29, 124003

Kotake, K., Sumiyoshi, K., Yamada, S., et al. 2012a, PTEP, 2012, 01A301

Kotake, K., Takiwaki, T., \& Harikae, S. 2012b, ApJ, 755, 84

Kotake, K., Yamada, S., \& Sato, K. 2005, ApJ, 618, 474

Kuroda, T., Kotake, K., \& Takiwaki, T. 2012, ApJ, 755, 11

Lattimer, J. M., \& Swesty, D. F. 1991, NuPhA, 535, 331

Lentz, E. J., Mezzacappa, A., Messer, O. E. B., Hix, W. R., \& Bruenn, S. W. 2012a, ApJ, 760, 94

Lentz, E. J., Mezzacappa, A., Messer, O. E. B., et al. 2012b, ApJ, 747, 73

Liebendörfer, M., Messer, O. E. B., Mezzacappa, A., et al. 2004, ApJS, 150,263

Liebendörfer, M., Rampp, M., Janka, H., \& Mezzacappa, A. 2005, ApJ, 620,840

Liebendörfer, M., Rosswog, S., \& Thielemann, F.-K. 2002, ApJS, 141, 229

Liebendörfer, M., Whitehouse, S. C., \& Fischer, T. 2009, ApJ, 698, 1174

Lindquist, R. W. 1966, AnPhy, 37, 487

Livne, E., Burrows, A., Walder, R., Lichtenstadt, I., \& Thompson, T. A. 2004, ApJ, 609, 277

Marek, A., \& Janka, H. 2009, ApJ, 694, 664

Melson, T., Janka, H.-T., Bollig, R., et al. 2015, ApJL, 808, L42

Mezzacappa, A., \& Bruenn, S. W. 1993a, ApJ, 405, 669

Mezzacappa, A., \& Bruenn, S. W. 1993b, ApJ, 410, 740

Mezzacappa, A., \& Bruenn, S. W. 1993c, ApJ, 405, 637

Mezzacappa, A., \& Messer, O. E. B. 1999, JCoAM, 109, 281

Mihalas, D., \& Mihalas, B. W. 1984, Foundations of Radiation Hydrodynamics (New York: Oxford Univ. Press)

Mösta, P., Richers, S., Ott, C. D., et al. 2014, ApJL, 785, L29

Müller, B., \& Janka, H.-T. 2014, ApJ, 788, 82

Müller, B., Janka, H.-T., \& Dimmelmeier, H. 2010, ApJS, 189, 104

Müller, B., Janka, H.-T., \& Heger, A. 2012a, ApJ, 761, 72

Müller, B., Janka, H.-T., \& Marek, A. 2012b, ApJ, 756, 84

Murphy, J. W., \& Burrows, A. 2008, ApJ, 688, 1159

Nagakura, H., Sumiyoshi, K., \& Yamada, S. 2014, ApJS, 214, 16

Nakamura, K., Kuroda, T., Takiwaki, T., \& Kotake, K. 2014, ApJ, 793, 45

Obergaulinger, M., Janka, H.-T., \& Aloy, M. A. 2014, MNRAS, 445, 3169

O'Connor, E. 2015, ApJS, 219, 24

O'Connor, E., \& Ott, C. D. 2010, CQGra, 27, 114103

O'Connor, E., \& Ott, C. D. 2011, ApJ, 730, 70

O'Connor, E., \& Ott, C. D. 2013, ApJ, 762, 126

Ott, C. D., Abdikamalov, E., Mösta, P., et al. 2013, ApJ, 768, 115

Ott, C. D., Burrows, A., Dessart, L., \& Livne, E. 2008, ApJ, 685, 1069

Pan, K.-C., Liebendörfer, M., Hempel, M., \& Thielemann, F.-K. 2015, ApJ, 817,72

Perego, A., Gafton, E., Cabezón, R., Rosswog, S., \& Liebendörfer, M. 2014a, A\&A, 568, A11

Perego, A., Hempel, M., Fröhlich, C., et al. 2015, ApJ, 806, 275

Perego, A., Rosswog, S., Cabezón, R. M., et al. 2014b, MNRAS, 443, 3134

Peres, B., Oertel, M. \& Novak, J. 2013, PhRvD, 87, 043006

Pons, J. A., Ibáñez, J. M., \& Miralles, J. A. 2000, MNRAS, 317, 550

Raffelt, G. G. 2001, ApJ, 561, 890

Rampp, M., \& Janka, H.-T. 2002, A\&A, 396, 361

Richers, S., Kasen, D., O'Connor, E., Fernández, R., \& Ott, C. D. 2015, ApJ, 813,38

Roberts, L. F., Shen, G., Cirigliano, V., et al. 2012, PhRvL, 108, 061103

Rosswog, S. 2009, NewAR, 53, 78

Rosswog, S. 2015a, MNRAS, 448, 3628

Rosswog, S. 2015b, IJMPD, 24, 30012

Rosswog, S., \& Liebendörfer, M. 2003, MNRAS, 342, 673

Rosswog, S., Piran, T., \& Nakar, E. 2013, MNRAS, 430, 2585

Ruffert, M., \& Janka, H. 1999, PThPS, 136, 287

Ruffert, M., Janka, H.-T., \& Schaefer, G. 1996, A\&A, 311, 532

Sanders, R., Morano, E., \& Druguet, M.-C. 1998, JCoPh, 145, 511

Scheck, L., Kifonidis, K., Janka, H.-T., \& Müller, E. 2006, A\&A, 457, 963

Scheidegger, S., Käppeli, R., Whitehouse, S. C., Fischer, T., \& Liebendörfer, M. 2010, A\&A, 514, A51

Sekiguchi, Y. 2010, COGra, 27, 114107

Sekiguchi, Y. 2011, JPhCS, 314, 012076

Sekiguchi, Y., Kiuchi, K., Kyutoku, K., \& Shibata, M. 2015, PhRvD, 91, 064059

Shapiro, S. L., \& Teukolsky, S. A. (ed.) 1986, Black Holes, White Dwarfs and Neutron Stars: The Physics of Compact Objects (Wiley)

Shibata, M., \& Taniguchi, K. 2011, LRR, 14, 6 
Sumiyoshi, K., Takiwaki, T., Matsufuru, H., \& Yamada, S. 2015, ApJS, 216, 5

Sumiyoshi, K., \& Yamada, S. 2012, ApJS, 199, 17

Sumiyoshi, K., Yamada, S., Suzuki, H., et al. 2005, ApJ, 629, 922

Suwa, Y. 2014, PASJ, 66, L1

Suwa, Y., Kotake, K., Takiwaki, T., Liebendörfer, M., \& Sato, K. 2011, ApJ, 738,165

Suwa, Y., Takiwaki, T., Kotake, K., et al. 2013, ApJ, 764, 99

Suwa, Y., Takiwaki, T., Kotake, K., \& Sato, K. 2007, PASJ, 59, 771

Suwa, Y., Yoshida, T., Shibata, M., Umeda, H., \& Takahashi, K. 2015, MNRAS, 454, 3073

Swesty, F. D., \& Myra, E. S. 2009, ApJS, 181, 1

Takiwaki, T., \& Kotake, K. 2011, ApJ, 743, 30

Takiwaki, T., Kotake, K., \& Sato, K. 2009, ApJ, 691, 1360
Takiwaki, T., Kotake, K., \& Suwa, Y. 2014, ApJ, 786, 83

Tamborra, I., Hanke, F., Müller, B., Janka, H.-T., \& Raffelt, G. 2013, PhRvL, 111,121104

Thompson, T. A., Burrows, A., \& Pinto, P. A. 2003, ApJ, 592, 434

Toro, E. F. 1997, Riemann Solvers and Numerical Methods for Fluid Dynamics. A Practical Introduction (Dordrecht: Springer-Verlag GmbH)

van Riper, K. A., \& Lattimer, J. M. 1981, ApJ, 249, 270

Winteler, C., Käppeli, R., Perego, A., et al. 2012, ApJL, 750, L22

Woosley, S. E., \& Heger, A. 2007, PhR, 442, 269

Woosley, S. E., Heger, A., \& Weaver, T. A. 2002, RvMP, 74, 1015

Yakunin, K. N., Marronetti, P., Mezzacappa, A., et al. 2010, CQGra, 27, 194005

Zhang, W., Howell, L., Almgren, A., et al. 2013, ApJS, 204, 7 